\title{
Rational engineering of xylanase hyper-producing system in Trichoderma reesei for efficient biomass degradation
}

\author{
Su Yan, Yan Xu and Xiao-Wei Yu*
}

\begin{abstract}
Background: Filamentous fungus Trichoderma reesei has been widely used as a workhorse for cellulase and xylanase productions. Xylanase has been reported as the crucial accessory enzyme in the degradation of lignocellulose for higher accessibility of cellulase. In addition, the efficient hydrolysis of xylan needs the co-work of multiple xylanolytic enzymes, which rise an increasing demand for the high yield of xylanase for efficient biomass degradation.

Results: In this study, a xylanase hyper-producing system in T. reesei was established by tailoring two transcription factors, XYR1 and ACE1, and homologous overexpression of the major endo-xylanase XYNII. The expressed xylanase cocktail contained $5256 \mathrm{U} / \mathrm{mL}$ xylanase activity and $9.25 \mathrm{U} / \mathrm{mL} \beta$-xylosidase ( $p N P X a s e$ ) activity. Meanwhile, the transcription level of the xylanolytic genes in the strain with XYR1 overexpressed was upregulated, which was well correlated with the amount of XYR1-binding sites. In addition, the higher expression of associated xylanolytic enzymes would result in more efficient xylan hydrolysis. Besides, 2310-3085 U/mL of xylanase activities were achieved using soluble carbon source, which was more efficient and economical than the traditional strategy of xylan induction. Unexpectedly, deletion of ace 1 in C30OExyr1 did not give any improvement, which might be the result of the disturbed function of the complex formed between ACE1 and XYR1. The enzymatic hydrolysis of alkali pretreated corn stover using the crude xylanase cocktails as accessory enzymes resulted in a 36.64\% increase in saccharification efficiency with the ratio of xylanase activity vs FPase activity at 500, compared to that using cellulase alone.

Conclusions: An efficient and economical xylanase hyper-producing platform was developed in T. reesei RUT-C30. The novel platform with outstanding ability for crude xylanase cocktail production would greatly fit in biomass degradation and give a new perspective of further engineering in T. reesei for industrial purposes.
\end{abstract}

Keywords: ACE1, Lignocellulose, Trichoderma reesei, Xylanase, Xylanolytic enzymes, XYNII, XYR1, Saccharification

\section{Background}

Renewable resource lignocellulose consists of cellulose $(40-60 \%)$ and hemicellulose (20-40\%) [1], which shows significant importance for bioresource conversion and sustainable development. The biodegradation of lignocellulose for biofuel production needs the enzymatical

*Correspondence: yuxw@jiangnan.edu.cn

Key Laboratory of Industrial Biotechnology, Ministry of Education, School of Biotechnology, Jiangnan University, Wuxi 214122, People's Republic of China hydrolysis that was mainly functioned by cellulase and hemicellulase. Several studies have been conducted to improve the hydrolysis efficiency by higher cellulase load and genetic manipulation of the cellulase producing strains for higher cellulase output [2,3]. However, after pretreatment, resident hemicellulose (mostly xylan) could form a sheath on each cellulose microfibril in lignocellulose $[4,5]$ which makes a barrier for further hydrolysis [6]. Xylan is mainly composed of D-xylose, which is linked with $\beta$-1,4-glycosidic bonds, and other D-galactose and L-arabinose substituent groups, making

(c) The Author(s) 2021. This article is licensed under a Creative Commons Attribution 4.0 International License, which permits use, sharing, adaptation, distribution and reproduction in any medium or format, as long as you give appropriate credit to the original author(s) and the source, provide a link to the Creative Commons licence, and indicate if changes were made. The images or other third party material in this article are included in the article's Creative Commons licence, unless indicated otherwise in a credit line to the material. If material is not included in the article's Creative Commons licence and your intended use is not permitted by statutory regulation or exceeds the permitted use, you will need to obtain permission directly from the copyright holder. To view a copy of this licence, visit http://creativeco mmons.org/licenses/by/4.0/. The Creative Commons Public Domain Dedication waiver (http://creativecommons.org/publicdomain/ zero/1.0/) applies to the data made available in this article, unless otherwise stated in a credit line to the data. 
a complex hetero-polymeric structure [1]. The biodegradation of xylan requires a complex enzyme cocktail that is commonly named xylanase [7]. Xylanase is a group of xylanolytic enzymes consisting of endo-xylanase, $\beta$-xylosidase, $\alpha$-L-arabinofuranosidase, acetyl xylan esterase, and ferulic acid esterase, which decompose xylan into simple monosaccharide and xylooligosaccharides $[1,8]$, showing great potential in the feed, biorefinery, and pulp paper industry. Generally, the xylanase production is achieved by overexpression of endo-xylanases [9], which shows high catalyzing performance to the backbone of xylan. However, the unthorough de-branch of the side-chain group also hinders the cleavage efficiency of endo-xylanase $[1,10]$, which then influences the accessibility of cellulose, resulting in lower fermentable sugar yield for downstream biofuel production. Thus, it is wise to use xylanase cocktail for efficient biomass degradation, compared to addition of single kind of xylanase.

Filamentous fungus Trichoderma reesei was widely used as a workhorse for cellulase and xylanase fermentation. It is reported that $80.6 \mathrm{~g} / \mathrm{L}$ extracellular protein, mainly composed of cellulase and xylanase, was produced in T. reesei through rational engineering [11]. Moreover, the genomic and transcriptional data from $T$. reese $i$ showed the expression of multiple xylanolytic enzymes that belonged to different families and exhibited different cleave specificity to xylan [12-15], giving the potential to use $T$. reese $i$ as a host for xylanase production in industrial scale. The main xylanases secreted under induction conditions are endo-xylanase XYNI and XYNII, which belong to the GH11 family and account for $90 \%$ of the secreted xylanase. The GH11 endo-xylanases act on xylan backbone and randomly cleave between $\beta$-1,4-glycosidic bonds, which termed as true xylanase. Among them, XYNII showed higher catalytic activity and stability [16, 17], giving better performance in industrial applications. Traditionally, the production of xylanases in T. reesei was achieved using the xylan-based substrate as the carbon source. However, the high cost and insolubility of xylan make unsatisfied and non-economical xylanase production, hindering further industrial fermentation.

The induction and expression of xylanolytic genes in T. reesei were co-regulated through multiple transcription factors (TFs). The widely investigated TF was the negative regulator CRE1, which controlled carbon catabolite repression (CCR) and could abolish the induction of cellulase and xylanase with the existence of easy-utilized carbon sources such as glucose [18]. Moreover, the transcription activator XYR1 served as a global TF that tightly controls the induction of cellulase and xylanase through specific binding to a $\mathrm{GGC}(\mathrm{A} / \mathrm{T})_{3}$ motif in the promoter [19]. ACE1 serves as a negative factor for cellulase and xylanase production, deletion of ace1 leads to increased production of cellulase and xylanase [20]. Besides, XPP1 [21] and SxlR [22] were also shown as the negative TFs for a few xylanases (XYN1, XYN2, XYN5, BXL2), deletion of which would cause an increase in xylanase production.

The recombinant expression of xylanase in T. reesei has already been performed using different promoters including Pxyn2, Pegl1, Pcbh2, Pcbh1, and Ppdc, and achieved different results [23-25]. The strongest cellobiohydrolase I promoter Pcbh1 that was highly induced with cellulosic carbon source has been widely used for recombinant expression in $T$. reesei $[26,27]$, while de Faria et al. made a low recombinant xylanase production using Pcbh1 which might due to the low catalytic activity of xylanase and the degradation of foreign protein [24]. $\mathrm{Li}$ et al. made the highest xylanase activity of $9266 \mathrm{U} /$ $\mathrm{mL}$ through the homologous expression of native XYNII under the strong pyruvate decarboxylase $(p d c)$ promoter with a high concentration of glucose (7\%) [28]. However, the strategy of the recombinant expression of XYNII in T. reesei QM9414 (a carbon catabolite repressed strain) only increased the endo-xylanase activity, further application in xylan degradation and other application needs the addition of other associated xylanolytic enzymes. Therefore, it is requisite to build a xylanase hyper-producing system that could produce different kinds of xylanases for efficient xylan and biomass degradation.

In this study, we developed a strategy that combined not only regulation of two transcription factors, XYR1 and ACE1, to obtain higher expression of xylanolytic enzymes, but also the homologous expression of the major xylanase XYNII under the Pcbh1 promoter in $T$. reesei for improved production of endo-xylanase. The xylanase cocktail produced by the recombinant strain was rich in endo-xylanase, associated with enhanced xylanolytic enzymes to limit the side-chain inhibition of xylan. Besides, the recombinant $T$. reesei could also achieve higher xylanase production in other economical carbon sources such as lactose or glucose medium, suggesting a more economical-friendly and flexible strategy for xylanase production. Moreover, the optimal ratio of xylanase $v s$ cellulase for saccharification was also analyzed for higher saccharification efficiency. Our study would give a new perspective for efficient biomass degradation and meet the increased demand for biofuel production.

\section{Results}

\section{Effects of XYR1 overexpression and ace 1 disruption on xylanase production}

In $T$. reesei, the induction of xylanase was triggered by cellulose, xylan and its polysaccharides. Meanwhile, the expression of xylanolytic genes was also regulated by 
transcription factors. To enhance the expression of different xylanolytic genes and increase xylanase production, we choose two well-characterized transcription factors, XYR1 and ACE1, which act as an activator and repressor for cellulase and xylanase production, respectively. As intended, we perform a combined strategy that upregulates activator XYR1 and downregulates the repressor ACE1 through inserting XYR1 overexpression cassette into ace1 locus. Transformant with correct insertion into ace 1 locus was verified by PCR (Fig. 1b, c, lanes 2, 4, and 6) and termed as C30OExyr1 $\Delta$ ace1. Simultaneously, a transformant harbored XYR1 overexpression cassette in ace1 locus by single-crossover homologous recombination was also constructed and termed as C30OExyr1, leaving an intact ace1 in the chromosome (Fig. 1a, c, lanes 1,3 , and 5 ). The scheme for the transformants construct was indicated in Fig. 1a, b. The transformants both showed a similar growth trend compared to the parent strain on the PDA plate (data not shown). In addition, the copy number of $x y r 1$ cassette in the parent strain and two transformants were also verified by qPCR (Fig. 1d) with primer listed in Additional file 1: Table S3.

Then, the xylanase-producing performance of the transformants and parent strain was analyzed through culturing three strains in Avicel-inducing medium. The result shows that the xylanase activity in C30OExyr1 and C30OExyr $1 \Delta$ ace1 is significantly increased compared to that of RUT-C30 (Fig. 2a). In addition, the highest xylanase activity of $1095 \mathrm{U} / \mathrm{mL}$ was achieved in C30OExyr1 on day 5, which was 1.62-fold higher than that of RUTC30 (Fig. 2a). Meanwhile, for further investigation of the xylanolytic enzymes, the $\beta$-xylosidase ( $p$ NPXase) activity was also analyzed. As shown in Fig. 2b, the $p$ NPXase activity in C30OExyr1 achieved $7.82 \mathrm{U} / \mathrm{mL}$ on day 5 , which was 2.29 -fold higher than that in RUT-C30. However, beyond expectation, the xylanase activity in C30OExyr1 $\triangle$ ace1 $(1015 \mathrm{U} / \mathrm{mL})$ on day 5 is a little bit lower than that of C30OExyr1 (1095 U/mL) (Fig. 2a). In
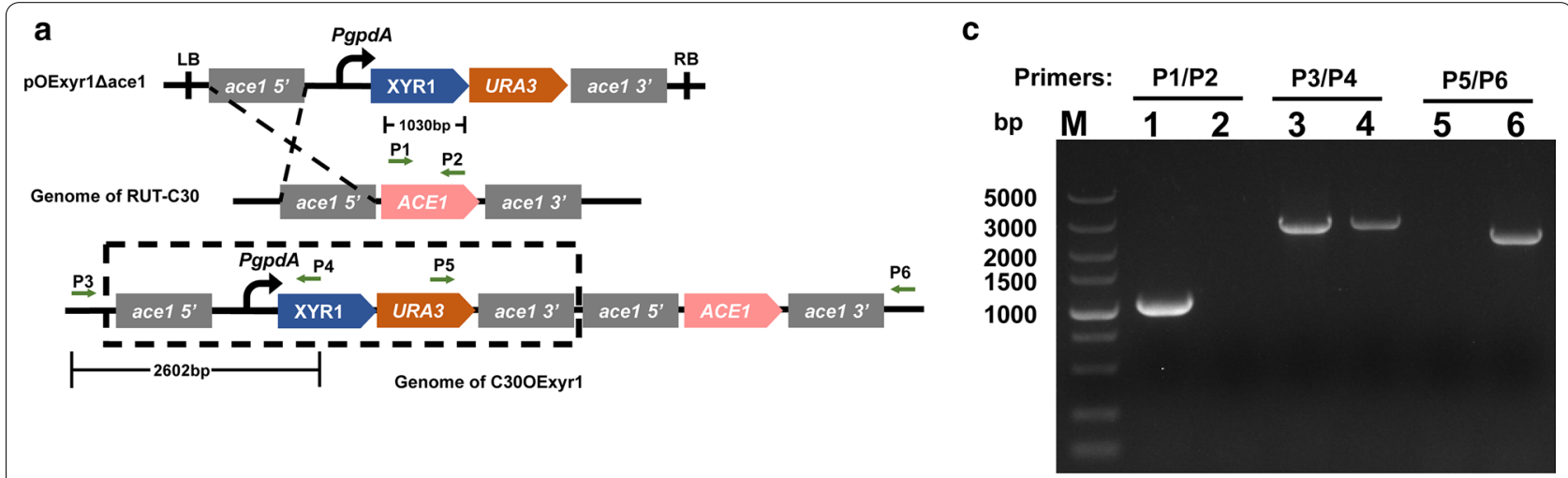

b

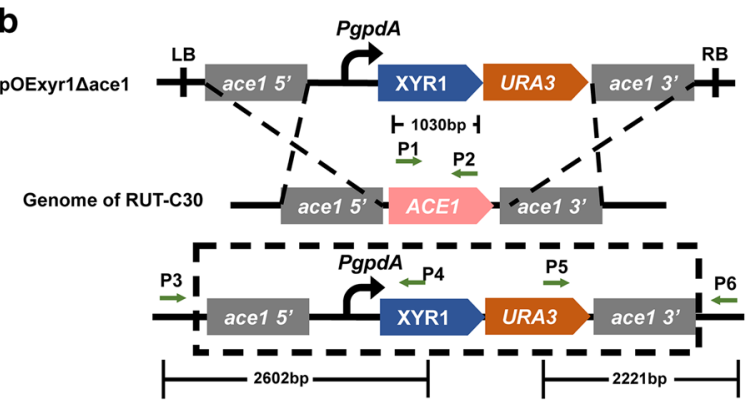

Genome of C30OExyr1 1 ace1 d

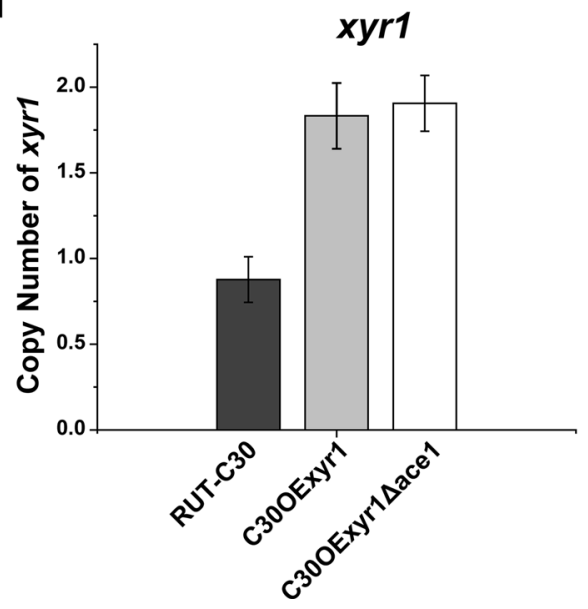

Fig. 1 The scheme for XYR1 overexpression and ACE1 disruption. a A scheme for C30OExyr1 construction. b A scheme for C30OExyr1 $\triangle$ ace 1 construction. c PCR analysis of recombinant C30OExyr1 and C30OExyr1 $\triangle$ ace 1. Lanes 1, 3, and 5 represent the PCR results of C30OExyr1, and Lanes 2, 4, and 6 represent the PCR results of C30OExyr1 $\triangle$ ace 1, which were detected using three pair primers indicated by arrows. $\mathbf{d}$ The copy number of xyr1 which was assayed by qPCR using the genomic DNA of RUT-C30, C30OExyr1 and C30OExyr1 $\triangle$ ace 1 as template, the gene sar 1 was represented as single copy 

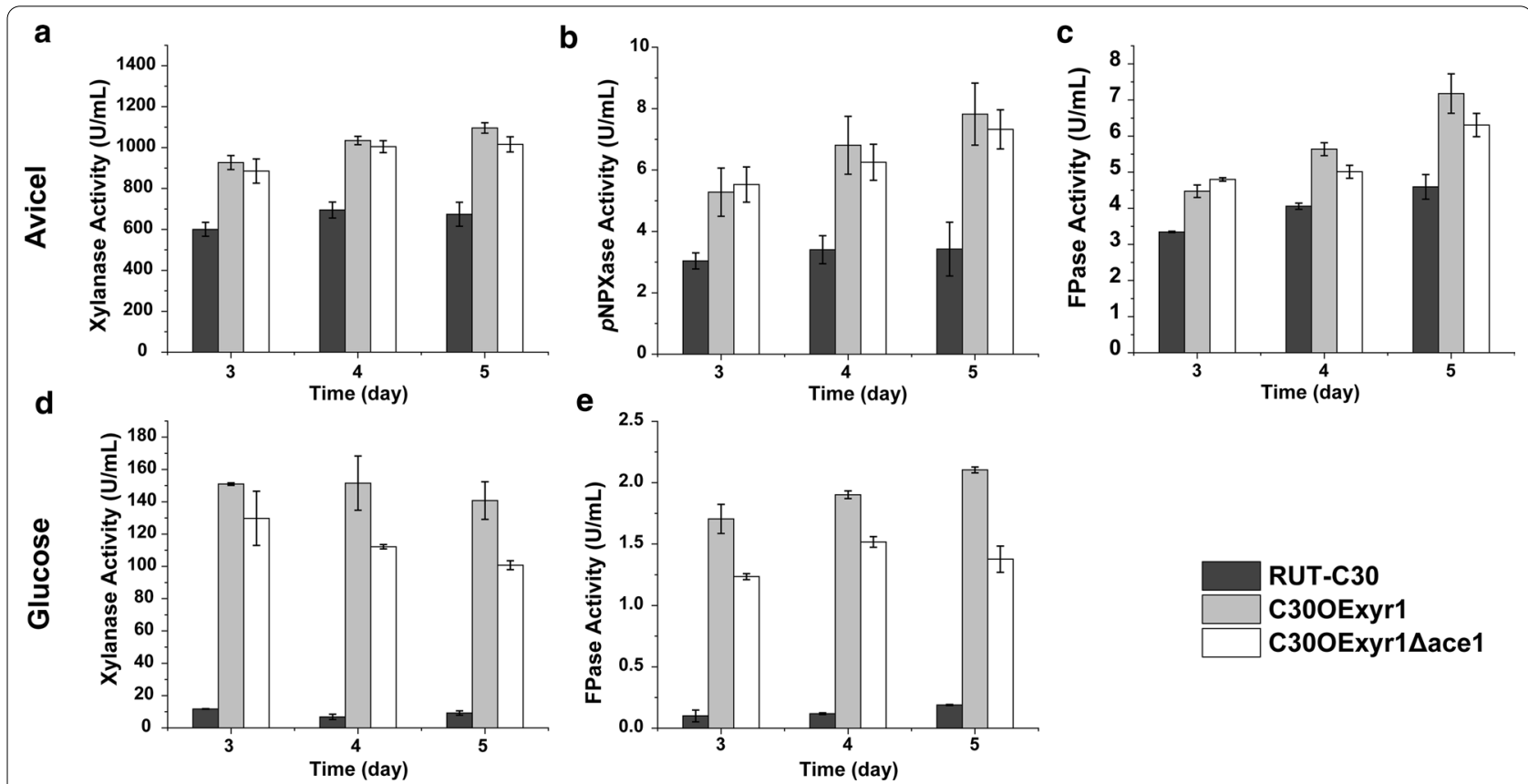

Fig. 2 Effects of XYR1 overexpression and ACE1 disruption on the FPase, xylanase and $p$ NPXase activities. a-c The xylanase $p N P X a s e$ and FPase activities of the recombinant strains RUT-C30, C30OExyr1 and C30OExyr1 $\triangle$ ace 1 cultured with 3\% Avicel. d, e The xylanase and FPase activities of RUT-C30, C30OExyr1 and C30OExyr1 $\triangle$ ace1 in 5\% glucose medium. For each experiment, three individual replicates were performed

addition, similar results were also attained for $p$ NPXase activity (Fig. 2b). Although the little discrepancy in our result did not show statistical significance, the biological repetition in different batch cultures was also conducted, which shows similar trends to the results in Fig. 2a, b, indicating that the ACE1 disruption in strain C30OExyr1 hardly give a positive effect as reported by Rauscher et al. [29]. Moreover, to test whether the unexpected result specifically affects xylanase and xylanolytic enzymes, we also test the cellulase activity as indicated by FPase activity. As shown in Fig. 2c, a similar result shows the ACE1 disruption in strain C30OExyr1 would give lower cellulase and xylanase output, rather than further enhance the production. Besides, to exclude the negative effect caused by ACE1 disruption in our study, C30 $\Delta$ ace 1 was also constructed and analyzed for cellulase and xylanase activity. Similar to the results achieved by Aro et al. [20], the cellulase and xylanase activity in C30 $\Delta$ ace 1 was higher than that in RUT-C30 (data not shown). The results show that the negative effect of ACE1 deletion is only present when XYR1 is overexpressed.

Furthermore, to test whether the decreased xylanase activity is carbon source dependent, the FPase and xylanase activities of C30OExyr1 $\Delta$ ace1, C30OExyr1, and their parent strain RUT-C30 were analyzed in a repression medium that glucose was used as the sole carbon source. As expected, the cellulase and xylanase activity in
RUT-C30 is almost repressed due to the CCR as reported previously [18] (Fig. 2d, e). While for C30OExyr1, the xylanase activity of $151.5 \mathrm{U} / \mathrm{mL}$ was achieved on day 4 due to the release of CCR, which was 13.7-fold higher than that of RUT-C30 (Fig. 2d). In addition, the cellulase and xylanase activity of C30OExyr1 was higher than that of C30OExyr1 $\triangle$ ace1 (Fig. 2d, e), which was similar to the trend in Avicel medium, suggesting that the negative effect of ace1 disruption while XYR1 overexpression is carbon source independent.

\section{Transcription profiles of xylanolytic genes in C300Exyr 1 and C30OExyr1 $1 \Delta$ ace 1}

To give a deep understanding of the decreased xylanase activity by ACE1 disruption with XYR1 overexpression, and to estimate the expression of other xylanolytic genes which contributed to better xylan utilization in two transformants C30OExyr1 1 ace 1 and C30OExyr1, seven xylanolytic genes were selected for further study. The analyzed xylanolytic genes include xyn1 (jgi|Trire2:74223), xyn2 (jgi|Trire2:123818) that encode for two major GH11 endo-xylanases, which were highly active on unbranched xylan backbone; xyn3 (jgi|Trire2:120229) that encodes for a GH10 endo-xylanase, prefer to hydrolyze short and branched xylan; xyn4 (jgi|Trire2:111849) encoding for GH30 xylanase harbored both exo- and endoxylanase activity; $b x l 1$ (jgi|Trire2:121127) encoding for 
$\beta$-xylosidase, act on xylooligosaccharides and release xylose residue; axe1 (jgi|Trire2:73632) and abf1 (jgi|Trire2:123283) that encode for acetyl xylan esterase and $\alpha$-L-arabinofuranosidase, respectively, which degrade side chain of xylan.

For transcription analysis, two transformants C30OExyr1 1 ace1, C30OExyr1 and their parent strain RUT-C30 were cultured in Avicel-inducing medium, and mycelia were sampled at $24 \mathrm{~h}$ and $48 \mathrm{~h}$ for analysis. First, the transcription level of xyrl was measured and the elevated transcription level of xyrl in
C30OExyr1 $\Delta$ ace1 and C30OExyr1 means the constitutively expressed XYR1 (Fig. 3c). Significantly, the transcription of $x y r 1$ of C30OExyr $1 \Delta$ ace 1 at $24 \mathrm{~h}$ was 2.19-fold higher than that in C30OExyr1 (Fig. 3c). Mach-Aigner et al. [30] demonstrated that the transcription of xyr1 was repressed by ACE1, which was highly similar to our result, indicating that higher transcription of xyr1 in this ACE1 disrupted transformant C30OExyr1 $\triangle$ ace1 might be attributed to the de-repression of ACE1. Meanwhile, the transcription level of ace1 was also determined, the result in Fig. 3c shows a

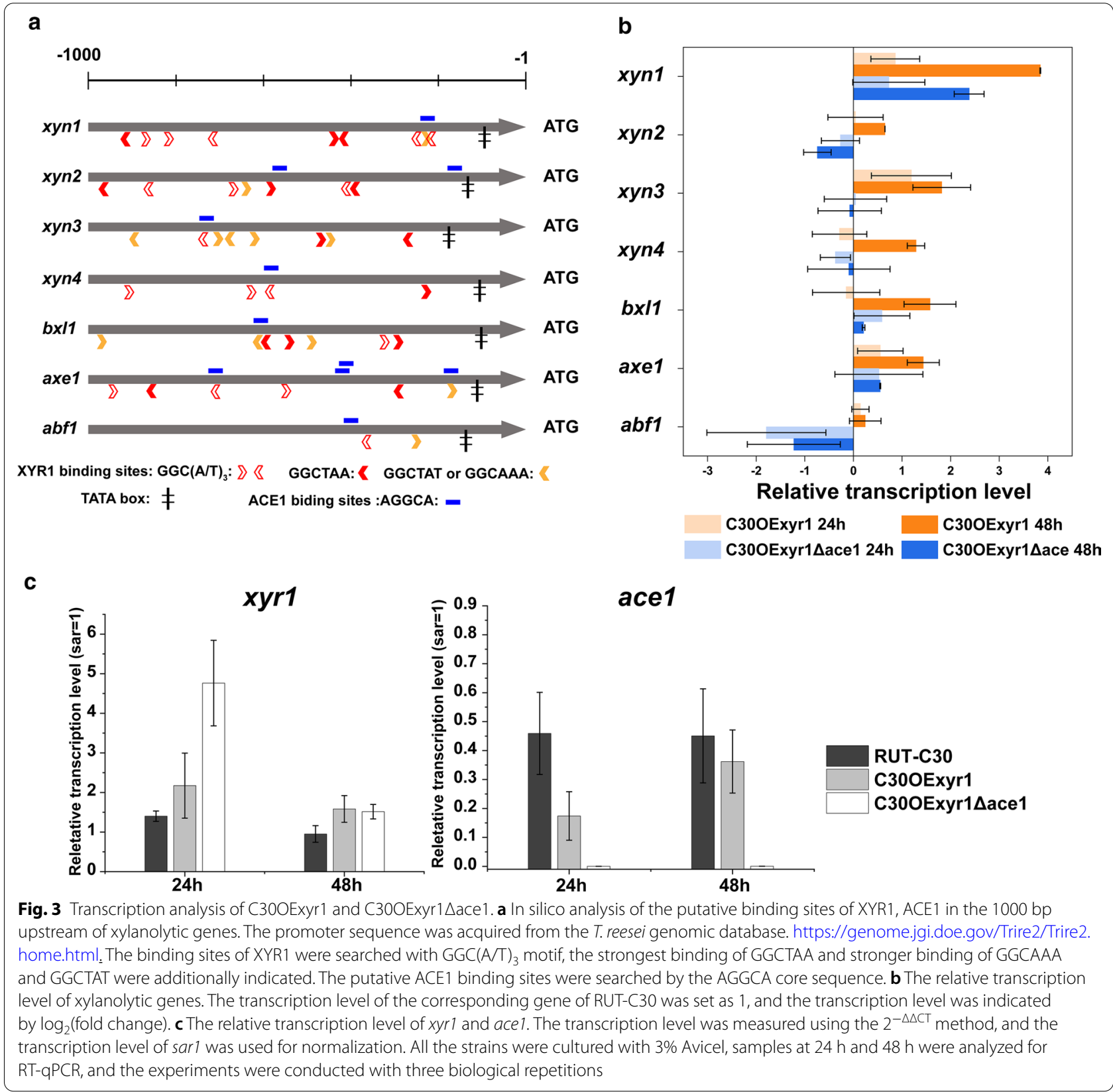


comparable transcription of ace1 in strain C30OExyr1 and RUT-C30 at $48 \mathrm{~h}$. The undetectable transcription of ace1 in C30OExyr1 1 ace1 further confirmed the complete loss of ACE1 function.

The analysis of the transcription level of seven xylanolytic genes demonstrated that the transcription of selected genes was highly upregulated in strain C30OExyr1 compared to that in C30OExyr1 1 ace 1 (Fig. 3b). Although higher transcription of xyrl could result in higher transcription of downstream genes [31], the higher transcription level of $x y r 1$ in C30OExyr1 $1 \Delta$ ace 1 no more give a higher transcription of downstream xylanolytic genes (Fig. 3b, c), suggesting that the loss of ACE1 function in C30OExyr1 might influence the regulation of XYR1.

For a detailed analysis of the enhanced transcription of xylanolytic genes, in silico analysis was also combined. We first explored the $1000 \mathrm{bp}$ upstream region of seven xylanolytic genes for the GGC(A/T) $)_{3}$ motif which represents the binding sites of regulator XYR1 [32], the motif before the TATA box is considered as the putative regulator binding sites of XYR1. As indicated in Fig. 3a, we identified nine XYR1-binding sites (XBD) in the $1000 \mathrm{bp}$ upstream of $x y n 1$. Among nine XBDs, three XBDs were reported as the best binding motif of XYR1 with the sequence of GGCTAA [32], and the preferable XBD GGCAAA was also detected in the promoter of $x y n 1$, which was well correlated with the 3.84-fold increase in the transcription level of $x y n 1$ at $48 \mathrm{~h}$ (Fig. 3b). Besides, the transcription level of $x y n 3, x y n 4, b x l 1$, and axe 1 was upregulated at least 1.28- to 1.81-fold in C30OExyr1 at $48 \mathrm{~h}$. In silico analysis revealed that from four to eight XBDs with a varied amount of GGCTAA/GGCTAT /GGCAAA motif was lied in the upstream region of these xylanolytic genes, indicating the strong transcription activation in strain C30OExyr1. Moreover, the lower transcription activation of abf1 in C30OExyr1 was consistent with fewer XBDs in its upstream region, and no GGCTAA motif was presented in this region, suggesting that $a b f 1$ was only weakly regulated by XYR1. However, the transcription of $x y n 2$ which encodes for the major endo-xylanase was only increased by 0.56 -fold at $48 \mathrm{~h}$, the less pronounced increase in $x y n 2$ transcription was not well correlated with seven XBDs with three GCC TAA motif in the upstream region (Fig. 3a, b). For strain C30OExyr1 $\Delta$ ace1 that lacks intact ace1, the upregulated transcription level of $x y n 1, b x l 1$ and axe 1 were detected both at $24 \mathrm{~h}$ and $48 \mathrm{~h}$, which was consistent with the enzyme activity data (Fig. 2a, b). While the transcription level of $x y n 2, x y n 3, x y n 4$ and $a b f 1$ was unchanged or downregulated compared to RUT-C30. Although several ACE1 binding sites were also detected in these xylanolytic genes, the transcription data did not show any correlation between transcription strength and the number of binding sites.

\section{Further enhancing xylanase production by XYNII overexpression}

In strain C30OExyr1, the transcription level of xyn1, $x y n 3, x y n 4, b x l 1$, and axe 1 were upregulated which lead to enhanced xylanase production in Avicel induction condition, however, the transcription level of major endoxylanase $x y n 2$ was only increased 0.56 -fold (Fig. 3b). In general, the GH11 endo-xylanase was termed as true xylanase due to their high catalytic ability towards xylan backbone [10], suggesting its significant role in xylan degradation. Among that, the specific activity of XYNII was 16-fold than that of XYNI [16], showing potential for xylan degradation. To further increase the capacity to cleave xylan backbone, we use the cellulose-induced promoter Pcbh1 [26, 27], which was the strongest promoter in $T$. reesei, to increase the production of endo-xylanase. The XYNII expression cassette was constructed as described in "Methods", and the cassette was transformed into C30OExyr1, C30OExyr1 1 ace1 and RUT-C30 to compare the xylanase-producing ability in the different strains. Meanwhile, for higher production, we also use a cassette that harbors $c b h 1$ homologous arms for sitedirected insertion in the $c b h 1$ locus, resulting in 6 recombinants for further analysis (Fig. 4a).

Xylanase production of different strains was performed by induction with $3 \%$ Avicel. The result shows that the xylanase activity in all transformants was significantly higher than that of RUT-C30. As expected, the transformants with a cassette inserted into cbh1 locus by homologous recombinant could produce higher xylanase activity than the transformants with random insertion (Fig. 4b), which was consistent with the previous study [33]. The higher XYNII output and the absence of CBHI were also verified by SDS-PAGE (Fig. 4d). The transformant C30/ xyn $2 \Delta$ cbh1, that the XYNII expression cassette was inserted into cbh1 locus in RUT-C30, achieved $3069 \mathrm{U} /$ $\mathrm{mL}$ xylanase activity after 5 day's induction, which was 4.33-fold higher than that in RUT-C30, revealing a dramatic increase in xylanase activity due to the overexpression of XYNII. The highest xylanase activity of $5256 \mathrm{U} /$ $\mathrm{mL}$ was achieved by C30OExyr $1 / \mathrm{xyn} 2 \Delta \mathrm{cbh} 1$ at day 4 , which was 6.79- and 1.71-fold higher compared to that of RUT-C30 and C30/xyn2 $\Delta$ cbh1, respectively (Fig. 4b). Similar to the result presented in Fig. 2, the transformant C30OExyr1 $\Delta$ ace $1 / x y n 2 \Delta$ cbh 1 produced much less xylanase activity than C30OExyr1/xyn2 $\Delta$ cbh1 (Fig. 4b). Moreover, the transcription level of $x y n 2$ was also measured in three strains. Results show that the transcription level of $x y n 2$ in C30OExyr1/xyn2 $\Delta$ cbh1 was higher than that in C30OExyr1 1 ace $1 / x y n 2 \Delta$ cbh 1 and C30/ 


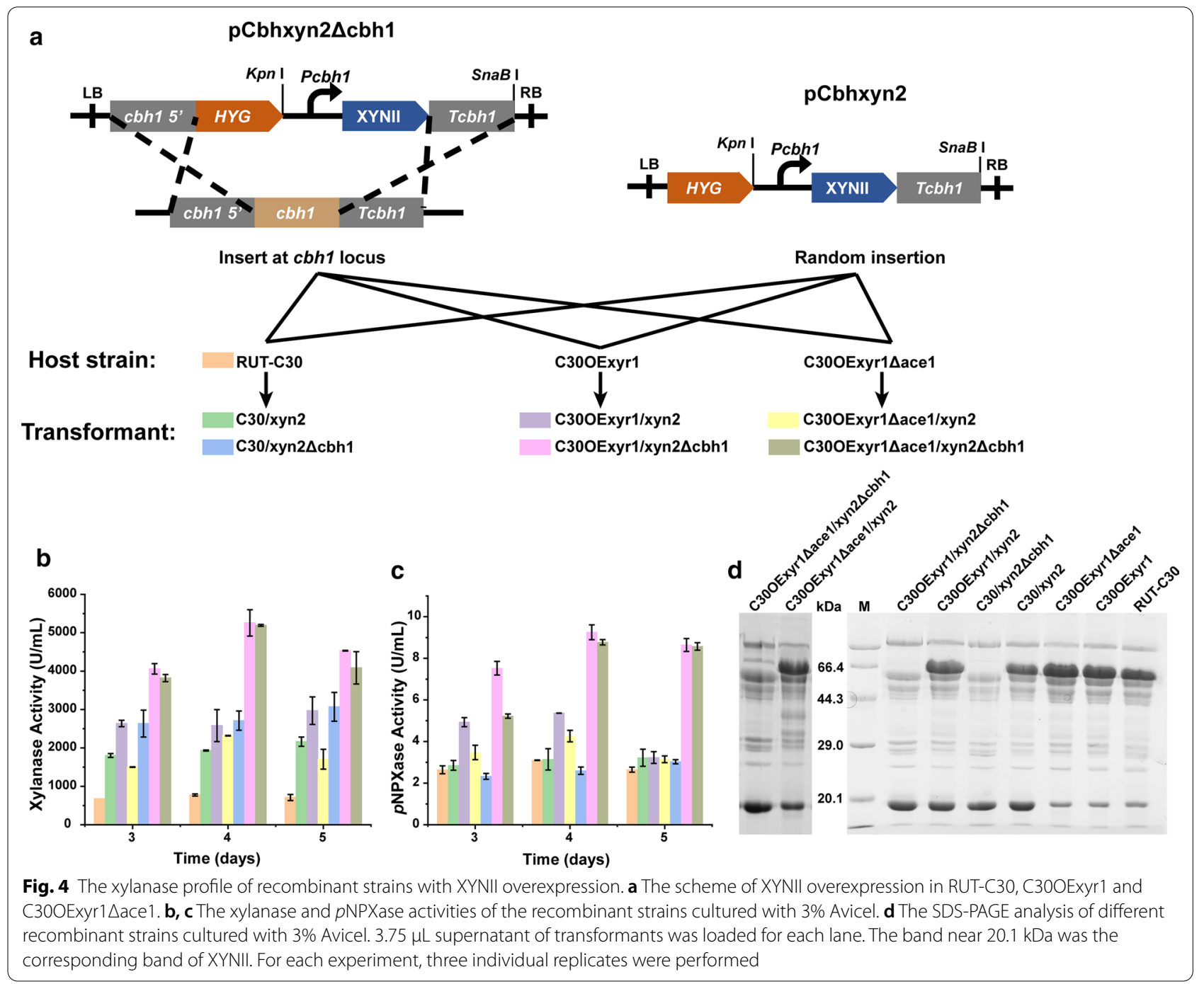

xyn $2 \Delta$ cbh1 either at $24 \mathrm{~h}$ or $48 \mathrm{~h}$ (Fig. 5b), which was consistent with the xylanase activity.

For analyzing other xylanolytic enzymes, the $p$ NPXase activity was also detected. As indicated in Fig. 4c, comparative $p$ NPXase activity was achieved in strains C30/ xyn2, C30/xyn2 $\Delta$ cbh 1 and RUT-C30. The $p$ NPXase activity in other transformants was much higher than that in RUT-C30 due to the overexpression of XYR1. In addition, $9.25 \mathrm{U} / \mathrm{mL} p$ NPXase activity was achieved in C30OExyr1/xyn2 $\Delta$ cbh1, which was about 2.98-fold higher than that of RUT-C30 (Fig. 4c). Not only that, the $p$ NPXase activity achieved in C30OExyr $1 /$ xyn $2 \Delta$ cbh 1 $(9.25 \mathrm{U} / \mathrm{mL})$ was higher than that in C30OExyr1 $(7.82$ $\mathrm{U} / \mathrm{mL}$ ) (Figs. 2b, 4c), suggesting that the transformant C30OExyr1/xyn2 $\Delta$ cbh1 could produce more abundant xylanolytic enzymes that were preferable for xylan degradation. Meanwhile, the transcription analysis of xylanolytic gene in C30OExyr1/xyn $2 \Delta$ cbh 1 and
C30OExyr1 $\Delta$ ace1/xyn2 $\Delta$ cbh1 also shows the upregulation of seven selected xylanolytic genes (Fig. 5a), especially in $x y n 1$ and $x y n 2$, which means a significant increase in GH11 endo-xylanase activity. Meanwhile, the transcription level of $x y n 3, b x l 1$, axe 1 and $a b f 1$ was also increased by at least onefold in C30OExyr1/xyn2 2 cbh1 (Fig. 5a), suggesting the upregulation of different xylanolytic genes. However, the transcription level of xyn4 was poorly activated in C30OExyr1/xyn2 2 cbh1 (Fig. 5a), which was inconsistent with the result in C30OExyr1 (Fig. 3b). Similar to the data in Fig. 3b, the transcription of xylanolytic genes above was weakly upregulated in C30OExyr1 1 ace $1 / x y n 2 \Delta$ cbh 1 than C30OExyr1/ xyn $2 \Delta$ cbh1, resulting in less pronounced xylanase-producing ability.

In summary, we use the strong cellulose-induced promoter Pcbh1 to increase the endo-xylanase activity of T. reesei. Compared to other strains constructed above, 

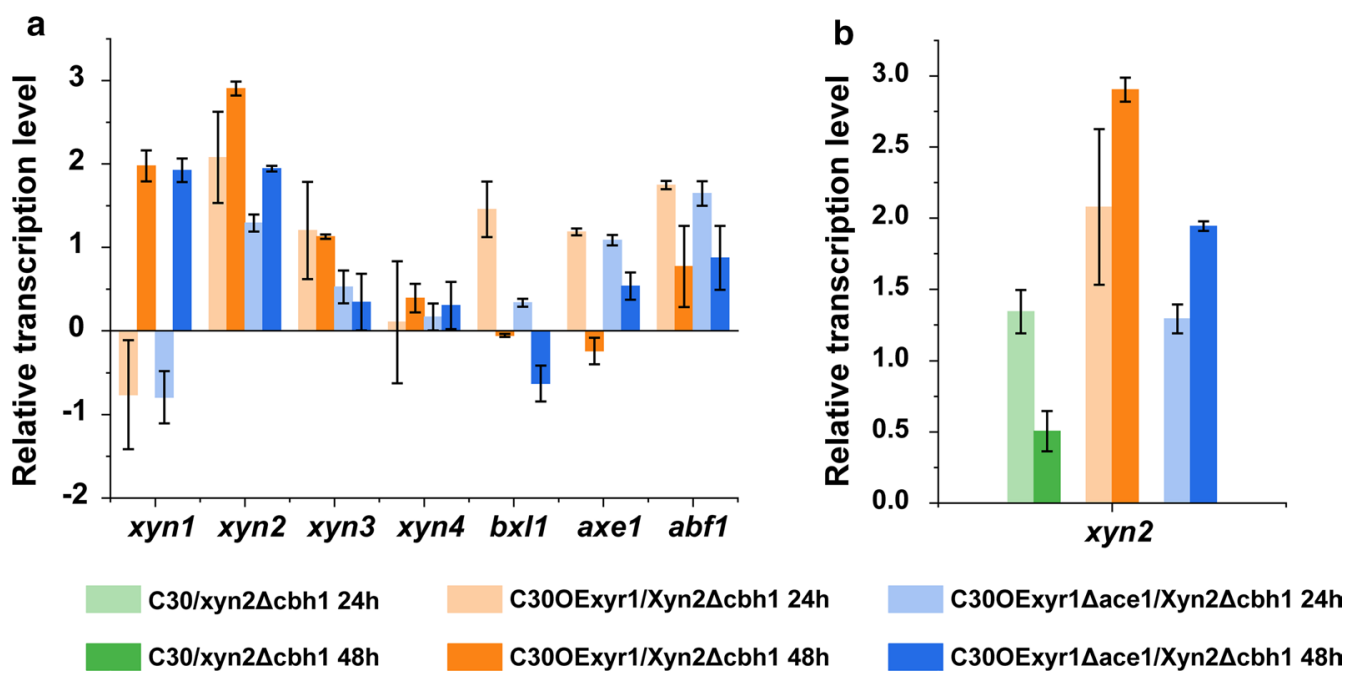

Fig. 5 Transcription level of xylanolytic genes in C30/xyn2 $\Delta$ cbh1, C30OExyr1/xyn2 $\Delta$ cbh1 and OExyr1 $\Delta$ ace $1 / x y n 2 \Delta c b h 1$. a The relative transcription level of xylanolytic genes in C30OExyr1/xyn2 $\Delta$ cbh1 and C30OExyr1 $\Delta$ ace 1/xyn2 $\Delta$ cbh1 cultured on Avicel. $\mathbf{b}$ The relative transcription level of $x y n 2$ in C30/xyn2 $\Delta$ cbh1, C30OExyr1/xyn2 $\Delta$ cbh1 and C30OExyr1 $\Delta$ ace1/xyn2 $\Delta \mathrm{cbh} 1$. The strains were all cultured in the Avicel medium and sampled at $24 \mathrm{~h}$ and $48 \mathrm{~h}$, respectively. The transcription level of the corresponding gene of RUT-C30 was set as 1, and the transcription level was indicated by $\log _{2}($ fold change). All the experiments were conducted with three biological replicates

the transformant C30OExyr1/xyn2 $\Delta$ cbh1 with XYNII expression at cbh1 locus gives the highest xylanaseproducing ability with strong expression of xylanolytic enzymes, showing potential for efficient biomass degradation. The xylanase production in different organisms was also compared (Additional file 1: Table S4), and the xylanase activity and $\beta$-xylosidase activity produced in our study were both prominent among other studies.

High-level xylanase production was achieved using soluble carbon source in C30OExyr1/xyn2 $2 \mathrm{cbh} 1$

Lactose and glucose were widely used as easily utilized, soluble and economical carbon sources for industrial fermentation. In T. reesei, lactose only moderately induces production of cellulase and xylanase [34] while glucose has been widely reported as a repressor due to CCR [18]. Then, in this section, the xylanase production was also assessed by culturing transformant C30OExyr1/ xyn $2 \Delta$ cbh 1 with lactose or glucose as sole carbon source, the xylanase activity and $p$ NPXase activity were detected as described in "Methods".

When lactose was used as the sole carbon source, the highest xylanase activity reached $3085 \mathrm{U} / \mathrm{mL}$ in C30OExyr1/xyn $2 \Delta \mathrm{cbh} 1$, and the $p$ NPXase activity was also increased to $1.41 \mathrm{U} / \mathrm{mL}$, which was 29.1-fold and 2.66-fold than that of RUT-C30 (Fig. 6a, b). When cultured with glucose as the carbon source, $2310 \mathrm{U} / \mathrm{mL}$ xylanase activity and $0.84 \mathrm{U} / \mathrm{mL} p$ NPXase activity was achieved in C30OExyr $1 / x y n 2 \Delta$ cbh 1 . While in parent strain RUT-C30, the xylanase production was mostly repressed, that $11 \mathrm{U} / \mathrm{mL}$ xylanase activity and $0.08 \mathrm{U} /$ $\mathrm{mL} p$ NPXase activity was detected, indicating a dramatic increase of xylanase production caused by the release of CCR. As well, the transcription level of xylanolytic genes was also measured, which shows an increased expression in xylanolytic enzymes either in lactose or glucose medium (Additional file 1: Figure S1), revealing that the xylanolytic enzymes could also be highly induced using lactose or glucose as carbon source. Glucose is an important carbohydrate that could participate in cellular metabolism, and the higher concentration of glucose could provoke higher transcription of pyruvate decarboxylase $(p d c)$ [28]. Li et al. achieved $9266 \mathrm{U} / \mathrm{mL}$ xylanase activity by constitutive expression of XYNII using this strong promoter $P p d c$. To compare the xylanase production of C30OExyr $1 / x y n 2 \Delta$ cbh 1 and the strain with constitutive expression of XYNII, we also constructed a strain using $P p d c$ promoter to initiate the transcription of $x y n 2$ and termed as C30/pdcxyn2. Unexpectedly, as indicated in Fig. 6a, the xylanase production of $1398 \mathrm{U} / \mathrm{mL}$ was achieved cultured with $5 \%$ glucose, which was much lower than that of C30OExyr $1 / x y n 2 \Delta$ cbh 1 in the same condition. Meanwhile, the $p$ NPXase activity of C30/ pdcxyn2 was also analyzed, showing complete repression as same as RUT-C30 (Fig. 6b).

In general, the induction of xylanase was performed using xylan as the inducer in $T$. reese $i$. Then, the xylanase production under xylan medium was also determined in the parent RUT-C30 by culturing strains with $3 \%$ xylan. It was obvious that the xylanase production 


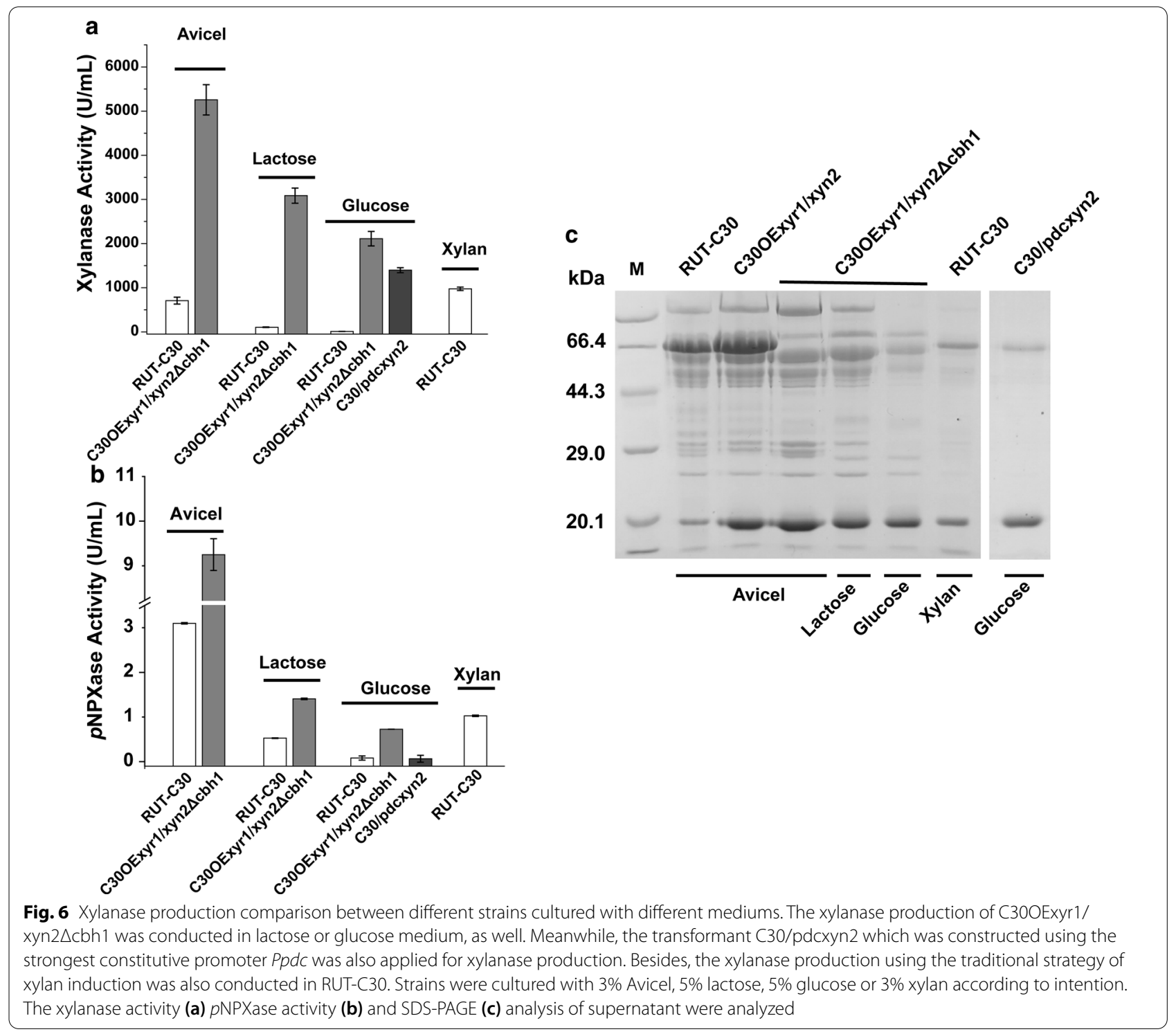

on xylan was higher than that on Avicel (Fig. 6a). However, the $p$ NPXase activity under xylan was nearly onethird of that on Avicel, suggesting the expression of $\beta$-xylosidase was more preferable on the cellulosic substrate. Besides, the xylanase production in C30OExyr1/ xyn $2 \Delta$ cbh 1 under different carbon sources was much higher than that of RUT-C30 cultured on xylan, which shows the better capacity for xylanase production in C30OExyr $1 / x y n 2 \Delta \mathrm{cbh} 1$. The SDS-PAGE analysis of xylanase production of different strains with different carbon was also conducted as indicated in Fig. 6c. The results show that the band near $20 \mathrm{kDa}$, referred to XYNII, was most abundant in C30OExyr $1 / x y n 2 \Delta$ cbh 1 than other strains discussed above.

\section{Synergistic effect with the addition of xylanase cocktails of C30OExyr $1 / x y n 2 \Delta \mathrm{cbh} 1$}

The addition of xylanase into cellulase, that termed as synergistic interaction, could improve the saccharification efficiency of biomass [6], resulting in higher reducing sugar yield. However, the addition of xylanase into the cellulase system for better saccharification requires further addition of enzyme, which increases the enzyme load and cost for saccharification (Table 1) [35, 36]. To systematically explore the appropriate rate of xylanase and cellulase in saccharification, we calculated the total cellulase and xylanase activity in each reaction as described in method section, and use a criterion $X / F$, the ratio of xylanase activity $v s$ FPase activity, to evaluate 
Table 1 Improvement of lignocellulose degradation by addition of xylanase in the literature

\begin{tabular}{|c|c|c|c|c|c|}
\hline \multirow[t]{2}{*}{ Biomass $^{\text {a }}$} & \multirow[t]{2}{*}{ Cellulase loaded without xylanase addition } & \multicolumn{2}{|l|}{ With xylanase addition } & \multirow[t]{2}{*}{ Improvement ${ }^{\mathrm{b}}(\%)$} & \multirow[t]{2}{*}{ Reference } \\
\hline & & Cellulase loaded & Xylanase loaded & & \\
\hline APCS & 8 mg/g biomass (12.2 FPU/g biomass) & $\begin{array}{l}5.48 \mathrm{mg} / \mathrm{g} \text { biomass ( } 8.4 \\
\mathrm{FPU} / \mathrm{g} \text { biomass) }\end{array}$ & $2.53 \mathrm{mg} / \mathrm{g}$ biomass & 34.64 & This study \\
\hline SPB & $2.1 \mathrm{mg} / \mathrm{g}$ biomass & $2 \mathrm{mg} / \mathrm{g}$ biomass & $0.1 \mathrm{mg} / \mathrm{g}$ biomass & 31.10 & [49] \\
\hline SPCS & $35 \mathrm{mg} / \mathrm{g}$ cellulose & $5 \mathrm{mg} / \mathrm{g}$ cellulose & $30 \mathrm{mg} / \mathrm{g}$ cellulose & 19.70 & {$[6]$} \\
\hline SPCS & $5 \mathrm{mg} / \mathrm{g}$ cellulose & $5 \mathrm{mg} / \mathrm{g}$ cellulose & $30 \mathrm{mg} / \mathrm{g}$ cellulose & $\sim 41$ & \\
\hline SPSB & & & & $\sim 31$ & \\
\hline SPLP & & & & $\sim 15$ & \\
\hline RTILs-PWS & $50 \mathrm{mg} / \mathrm{g}$ biomass & $50 \mathrm{mg} / \mathrm{g}$ biomass & $50 \mathrm{IU} / \mathrm{g}$ biomass & 56.71 & {$[50]$} \\
\hline APCS & $25 \mathrm{FPU} / \mathrm{g}$ biomass & $25 \mathrm{FPU} / \mathrm{g}$ biomass & $200 \mathrm{U} / \mathrm{g}$ biomass & $90.20^{c}$ & {$[36]$} \\
\hline APCS & $1.38 \mathrm{FPU} / \mathrm{g}$ biomass & $1.38 \mathrm{FPU} / \mathrm{g}$ biomass & $1.06 \mathrm{mg} / \mathrm{g}$ biomass & $55.60^{d}$ & {$[51]$} \\
\hline SPRS & $1 \mathrm{~mL}$ cellulase (sigma) & $1 \mathrm{~mL}$ cellulase (sigma) & $0.42 \mathrm{mg} / \mathrm{g}$ wet biomass & 12.40 & {$[35]$} \\
\hline
\end{tabular}

a APCS: alkali pretreated corn stover; SPB: alkaline pretreated bagasse; SPCS: steam pretreated corn stover; SPSB: steam pretreated sweet sorghum bagasse; SPLP: steam pretreated lodgepole pine; RTILs-PWS: RTILs (1-ethyl-3-methylimidazolium acetate) pretreated wheat straw; SPRS: steam pretreated rice straw

b The improvement means the improvement in released glucose with xylanase addition compared to that without xylanase addition

c The improvement of $90.2 \%$ was measured after $8 \mathrm{~h}$ saccharification, which released $14 \mathrm{mM}$ reducing sugar

d The improvement of $55.60 \%$ finally makes a $46.70 \%$ hydrolysis of cellulose

Table 2 The specific activity of enzyme preparation

\begin{tabular}{llllrl}
\hline & $\begin{array}{l}\text { Protein } \\
\text { concentration } \\
(\mathrm{mg} / \mathrm{mL})\end{array}$ & $\begin{array}{l}\text { FPase }(\mathrm{UL}) \\
\mathrm{mL})\end{array}$ & $\begin{array}{l}p N P G a s e \\
(\mathrm{U} / \mathrm{mL})\end{array}$ & $\begin{array}{l}\text { Xylanase } \\
(\mathrm{U} / \mathrm{mL})\end{array}$ & $\begin{array}{l}\text { pNPXase } \\
(\mathrm{U} / \mathrm{mL})\end{array}$ \\
\hline Cellulase $^{\mathrm{a}}$ & 2.56 & 3.93 & 3.72 & 93.93 & 0.78 \\
Xylanase $^{\mathrm{b}}$ & 2.22 & 1.57 & 1.80 & 4298.24 & 7.37 \\
\hline
\end{tabular}

a The commercial cellulase used in this study is purchased from Sunson (NingXia, China)

b The xylanase cocktail was produced by culturing C30OExyr1/xyn2 $2 \mathrm{cbh} 1$ in Avicel medium for 5 days

the amount of xylanase and cellulase in the saccharification system. The profile of enzyme preparation is listed in Table 2. The alkali pretreated corn stover (APCS) was used as substrate for saccharification and equal amount (protein concentration) of enzyme was loaded in each reaction, and a series of $X / F$ from 24 to 2770 were set as indicated in Table 3.

As expected, the result shows that the xylose content was continuously increased with the increasing proportion of xylanase. The highest xylan hydrolysis efficiency of $91.08 \%$ was achieved at the $X / F=2770$, giving the highest xylose yield of $9.48 \mathrm{mg} / \mathrm{mL}$ (Additional file 1: Table S5). With higher xylanase addition, the cellulase proportion and total cellulase activity were continuously decreased, while the glucose yield was significantly improved with appropriate xylanase addition (Fig. 7a, Table 3). In addition, the highest glucose yield (18.20 mg/ $\mathrm{mL}$ ), which was corresponding to the cellulose hydrolysis of $63.59 \%$, was reached with $X / F=500$, which was $34.64 \%$ higher than that using cellulase alone (Table 3, Additional
Table 3 The detailed content of enzyme preparation

\begin{tabular}{llllll}
\hline$X^{\prime} F^{a}$ & $\begin{array}{l}\text { Cellulase } \\
\text { volume } \\
(\mathrm{mL})\end{array}$ & $\begin{array}{l}\text { Total } \\
\text { FPase } \\
\text { activity } \\
(\mathrm{U})\end{array}$ & $\begin{array}{l}\text { Xylanase } \\
\text { volume } \\
(\mathrm{mL})\end{array}$ & $\begin{array}{l}\text { Total } \\
\text { xylanase } \\
\text { activity }(\mathrm{U})\end{array}$ & $\begin{array}{l}\text { Improvement } \\
(\%)\end{array}$ \\
\hline 24 & 1.56 & 6.14 & 0 & 146.53 & 100.00 \\
50 & 1.53 & 6.07 & 0.04 & 315.64 & $110.82 \pm 2.19$ \\
100 & 1.47 & 5.94 & 0.11 & 610.88 & $114.99 \pm 1.63$ \\
200 & 1.36 & 5.71 & 0.24 & 1159.32 & $126.14 \pm 3.91$ \\
500 & 1.07 & 5.09 & 0.57 & 2550.50 & $134.63 \pm 5.37$ \\
1000 & 0.70 & 4.32 & 0.99 & 4321.01 & $130.58 \pm 3.31$ \\
1500 & 0.43 & 3.75 & 1.30 & 5628.10 & $120.90 \pm 4.50$ \\
2000 & 0.23 & 3.31 & 1.54 & 6640.89 & $111.10 \pm 3.27$ \\
2770 & 0 & 2.83 & 1.80 & 7735.83 & $85.48 \pm 7.20$ \\
\hline
\end{tabular}

a $X / F$ means the xylanase activity vs FPase activity in the saccharification system. And equal amount of enzyme ( $8 \mathrm{mg} / \mathrm{g}$ APCS) was loaded for each reaction

b Improvement refers to the improved glucose yield compared to that using cellulase alone $(X / F=24)$

file 1: Table S5). At $X / F=500$, the cellulase addition was only $68.6 \%$ of that using cellulase alone, while the glucose yield gave a $34.64 \%$ increase, compared to that of $X / F=24$. Although further increased xylanase proportion could also improve glucose yield than that using cellulase alone $(X / F=24)$, the glucose yield was continuously reduced with $X / F$ beyond 500 (Fig. 7a, c). In addition, when $X / F$ reached 2000, the cellulase load was only $1.18 \mathrm{mg} / \mathrm{g}$ biomass, which was $14.75 \%$ of that using cellulase alone, and the total FPase activity for saccharification was $3.31 \mathrm{U}$, which was about $54 \%$ of that at $X / F=24$, 

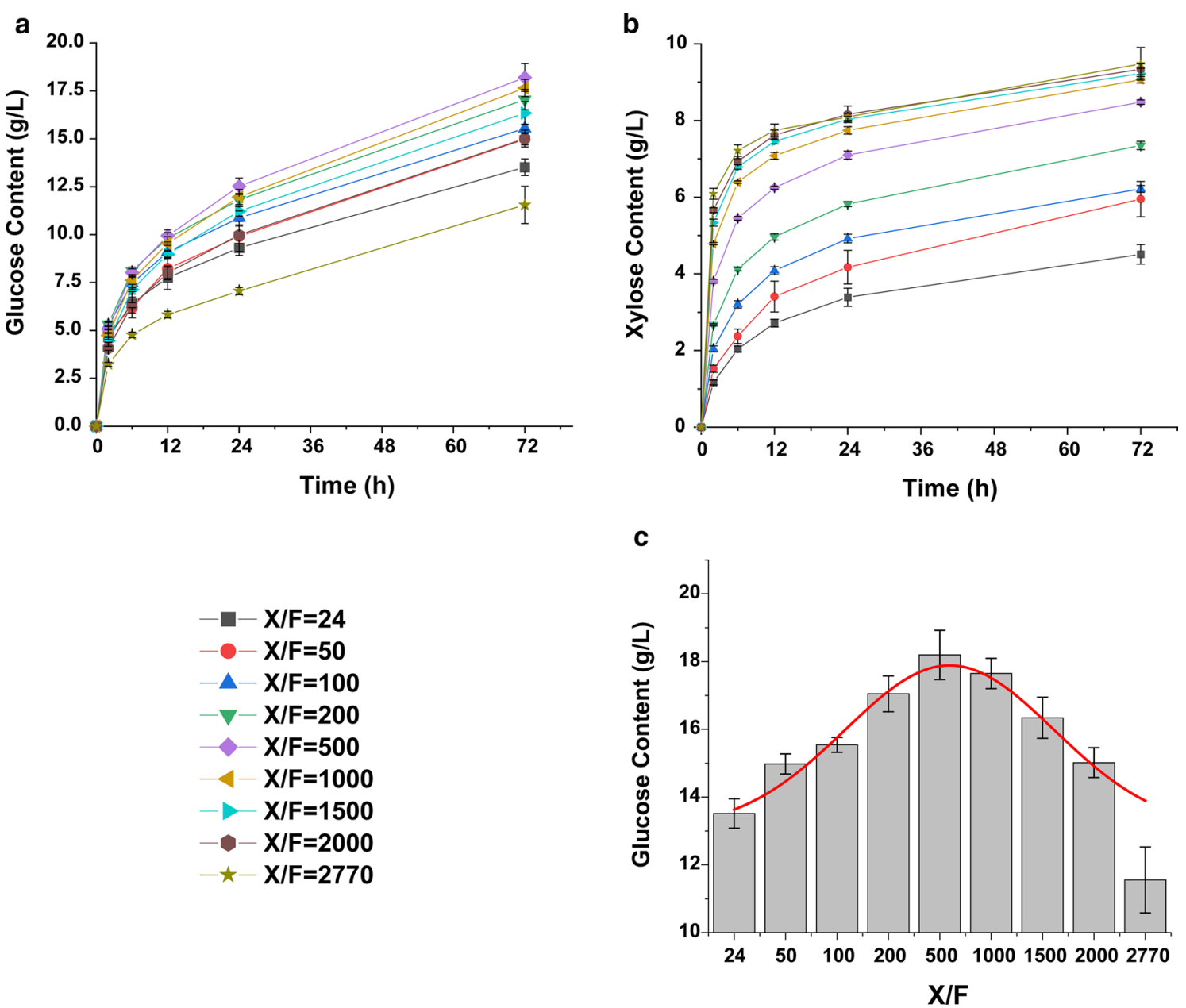

Fig. 7 Saccharification of APCS with xylanase cocktail produced by C30OExyr1/xyn2 $\triangle \mathrm{cbh} 1$ as the accessory enzyme. The glucose content (a) and xylose content (b) of different saccharification groups were analyzed by HPLC, an equal amount of enzyme load (8 mg/g APCS) was applied. The comparison of glucose content after $72 \mathrm{~h}$ hydrolysis was also presented (c). Three individual biological repeats were performed for each reaction

while the glucose yield was still 1.11-fold higher than that using cellulase alone (Fig. 7c, Table 3). Moreover, further increase in xylanase proportion gave a total 15\% decrease in glucose yield, which might be attributed to the unbalanced proportion of cellulase and xylanase.

\section{Discussion}

The crucial step of lignocellulose utilization for renewable resource production is to hydrolyze polysaccharides into fermentable sugar, and the degradation of lignocellulose requires the synergistic effect of cellulase and xylanase. However, xylanase was also reported as an ideal accessory enzyme for enhanced saccharification efficiency [37], which is due to the "xylanase boosting effect" [6] that breaks the crosslink between cellulose and xylan, and improves the accessibility of cellulase for better hydrolysis [6]. The hydrolysis of xylan was conducted with multiple xylanolytic enzymes [1], among that, the GH11 endo-xylanase was highly active on the backbone of xylan, but the efficient cleavage by endo-xylanase could also be impeded by the sub-chain group [10], which need the assistance of other xylanolytic enzymes. To establish a hyper-xylanase-producing platform, a series of efforts were conducted in $T$. reesei $[22,25]$, a potential fungus for the expression of multiple xylanolytic enzymes, to produce a highly active xylanase cocktail for xylan degradation.

In this study, first, the well-characterized transcription activator XYR1 and repressor ACE1 were manipulated. Beyond expectation, the combined strategy of upregulation of XYR1 and disruption of ACE1 resulted in slightly lower xylanase activity than that in C30OExyr1 (Fig. 2a), a transformant with XYR1 overexpression alone. ACE1 was identified as a repressor for cellulase production [20], and its deletion can increase the transcription level 
of cellulase and xylanase related gene. Moreover, a similar strategy conducted by Wang et al. [38] that combining XYR1 overexpression and ACE1 suppressing through RNAi resulted in higher xylanase and cellulase activities compared with sole XYR1 overexpression [38], which was opposite to our result. Besides, the expression of cellulase and xylanase was correlated with the abundance of XYR1 [31], and the expression of XYR1 in transformants could also be influenced by the locus of expression cassette and copy number. In our study, the copy number of xyr1 was kept as the same in C30OExyr1 and C30OExyr1 $\Delta$ ace1 (Fig. 1d), with the same insertion locus at the ace1 site (Fig. 1a, b). While the transformant with overexpressed XYR1 cassette was randomly selected by Wang et al. [38], which was difficult to exclude the discrepancy in insertion locus. Moreover, the transcription level of $x y r 1$ in C30OExyr1 1 ace1 was much higher than that in C30OExyr1 at 24 h (Fig. 3c), the result was consistent with the previous study that ACE1 could antagonize xyr1 transcription [30] through direct binding to the promoter of $x y r 1$ [39]. Besides, at $48 \mathrm{~h}$, the transcription level of $x y r 1$ was comparative in two transformants. Surprisedly, the higher or comparative transcription of xyr1 did not give a higher transcription of the xylanolytic gene in C30OExyr1 $\Delta$ ace1 (Fig. 3b). ACE1 was reported that participate in the regulation through forming a protein complex with XYR1 at GGCTAA motif [29]. Indeed, the transcription regulation through regulator was more likely to form a polymer. In Saccharomyces cerevisiae, the $\mathrm{Zn}(\mathrm{II})_{2} \mathrm{Cys}_{6}$ type transcription regulator GAL4 was more likely to act as a dimer [40]. In $T$. reesei, the inverse repeat of GGC(A/T $)_{3}$ was crucial for transcription of $x y n 3$ [41] and $x y n 1$ [29], suggesting that XYR1 could also functionally bind to the promoter as a homodimer. Meanwhile, the acidic activation domain of XYR1 was also verified for direct binding of ACE3 [42]. Thus, we speculated that in this study, the constitutive expression of XYR1 might disturb the distribution between XYR1 and ACE1, and further deletion of ACE1 could hinder the interaction between XYR1 and ACE1 which would lead to decreased production. In addition, the discrepancy between the two results of Wang et al. [38] and our study might be explained by that the suppression of ace1 through RNAi only attenuates ACE1 function, but not to eliminate it. Our results give a new perspective that ACE1 might also be involved in the functional regulation of XYR1, but the mechanism still needs to be further investigated.

The transcription analysis of xylanolytic genes shows upregulation in C30OExyr1 (Fig. 3b), and the significantly upregulated genes were well correlated with the distribution of XYR1-binding sites except for $x y n 2$ (Fig. 3a), the gene encoding for major GH11 endo-xylanase in $T$. reesei. Mach-Aigner et al. also reported a less pronounced increase in the transcription level of $x y n 2$ compared to $x y n 1$ [30], which was in agreement with our data. Besides, partially constitutive expression of xyn2 and other factors might also be involved in the direct regulation of xyn2 other than XYR1 [21, 43]. Then, a strategy for enhanced endo-xylanase activity was performed by overexpression XYNII with the strongest Pcbh1 promoter. Evidently, the $c b h 1$ locus was suitable for recombinant XYNII expression compared to the transformant with a randomly inserted cassette (Fig. 4b). Qin et al. show that the cbh1 locus was highly transcribed [33], which was preferable for recombinant protein expression. In addition, the transcription level of $x y n 2$ was higher in C30OExyr1/xyn2 $\Delta$ cbh1 than that in C30OExyr1/xyn2 (Additional file 1: Figure S2. B), suggesting the higher xylanase production in C30OExyr1/xyn $2 \Delta$ cbh 1 was partially due to the higher expression of XYNII in $c b h 1$ locus. Besides, the cassette inserted into $c b h 1$ locus with homologous recombination also resulted in the absence of major cellulase CBHI. CBHI was the most abundant cellulase under cellulose induction, which account for more than $50 \%$ of total secreted protein [44]. Theoretically, the absence of CBHI would leave more room for recombinant protein secretion, and release the ER stress for secreted protein [45]. In our study, improved xylanase production was also observed in CBHI deleted transformant (Fig. 4b) might due to the alleviation of feedback mechanism called RESS (REpression under Secretion Stress), which downregulates the transcription of cellulase and hemicellulase when suffered with ER stress [46]. In C30OExyr1/xyn2, decreased transcription of xyn1 and $b x l 1$ was detected compared to that of C30OExyr1/ xyn $2 \Delta$ cbh1 (Additional file 1: Figure S2. C), which probably result from the ER stress caused by recombinant XYNII expression, while in C30OExyr $1 / x y n 2 \Delta$ cbh 1 , ER stress was alleviated due to the absence of CBHI, which weaken the negative effect of RESS. We also used a CBHI deleted mutant $C 30 \Delta$ cbh1 to evaluate the feedback caused by released ER stress, the result also showed the increased $\beta$-xylosidase activity compared to that of the parent strain (Additional file 1: Figure S3). Moreover, ER stress could also trigger the activation of UPR (Unfolded Protein Response), while the detection of the transcription of UPR related gene shows a higher transcription level in C30OExyr1/xyn2 $\Delta$ cbh1 than that in C30OExyr1/ xyn2 (Additional file 1: Figure S2. A), which might be explained by the independent regulation pathway of UPR and RESS [47].

Meanwhile, the xylanase production in C30OExyr1/ xyn $2 \Delta$ cbh 1 was also applied using the economical and soluble carbon source lactose or glucose as the sole carbon source, and the xylanase production using lactose or glucose as carbon source was higher than that using xylan 
as an inducer (Fig. 6a). Li et al. [28] reported a relatively high XYNII activity of $9266 \mathrm{U} / \mathrm{mL}$ by constitutive overexpression of XYNII in T. reesei. In this study, we also overexpressed XYNII in T. reesei using $p d c$ promoter (C30/ pdcxyn2) according to $\mathrm{Li}$ et al. [28]. The xylanase production of C30/pdcxyn2 $(1398 \mathrm{U} / \mathrm{mL})$ was less than that of C30OExyr1/xyn2 $\Delta \mathrm{cbh} 1(2310 \mathrm{U} / \mathrm{mL})$ with glucose as carbon source. Moreover, the xylanase production in C30/pdcxyn2 was much lower than that achieved by $\mathrm{Li}$ et al. [28]. The fermentation medium used by Li et al. [28] was nutrient-rich which might result in higher xylanase output. Besides, the difference between transformants due to the insertion locus and copy number, and the substrate used for xylanase activity determined could also affect the final result. Obviously, in the same condition, C30OExyr1/xyn2 $\Delta$ cbh1 was preferable for xylanase production than C30/pdcxyn2 even in glucose medium. In addition, the lower xylanase production using lactose and glucose compared to that of Avicel could be conquered by fed-batch fermentation in a bioreactor, showing potential for applying on the industrial scale.

Afterward, the xylanase preparation with higher xylanase production achieved in C30OExyr $1 / x y n 2 \Delta \mathrm{cbh} 1$ was tested for its saccharification ability with cellulase. In our study, a ratio of xylanase activity vs FPase activity $(X / F)$ was set as a parameter to estimate the proper proportion of xylanase and cellulase under the same enzyme load. With higher proportion of xylanase, the xylan was hydrolyzed rapidly at early stage, improving accessibility for cellulase. However, with the same enzyme loaded, the proportion of cellulase and xylanase was critical for better saccharification. As shown in the result, the highest glucose yield was achieved at $X / F=500$ under the same enzyme load (Fig. 7c). Besides, it was interesting that using a small amount of cellulase to improve saccharification with the higher xylanase proportion at $X / F=2000$ (Fig. 7c), the efficient hydrolysis of xylan (Fig. 7b) loosen the complex structure of lignocellulose, and then, less amounts of cellulase could easily bind to the cellulose structure and make saccharification more efficient than that using cellulase alone, as indicated by $\mathrm{Hu}$ et al. [6]. Besides, the higher $\beta$-xylosidase activity in saccharification could attenuate inhibition of cellulase and xylanase activity by the decreased accumulation of xylooligosaccharides [48], showing potential for biomass conversion. Therefore, our study also provides a new strategy to keep the appropriate amount of xylanase cocktail to optimize the enzyme load for efficient lignocellulose saccharification. In this study, a significant increase in glucose yield was present with the increasing proportion of xylanase, which was partially attributed to the higher hemicellulose content (23.4\%) in the pretreated lignocellulose. Liu et al. also reported increased saccharification of hemicellulose-rich corn straw by increase the abundance of XYNII through deletion of regulator SxlR [22], and $\mathrm{Hu}$ et al. also certified the decreased improvement of saccharification efficiency with the reduction of xylan content in different substrates [6]. However, due to different substrate characteristic and the different pretreated strategy, the hemicellulose content of different biomass was varied, so it was difficult to say whether the best ratio of $X / F=500$ was also suitable for other biomass saccharification, but our work present here could also provide an effective strategy for higher xylanase production, which shows excellent performance in the saccharification of hemicellulose-rich lignocellulose. Besides, the comparison of saccharification results in different studies using xylanase addition also shows that our result gives a higher improvement in saccharification efficiency with the same enzyme load $[6,49]$. Although higher improvement has also been achieved [36, 50, 51], further addition of xylanase with the same cellulase load increases costs (Table 1).

\section{Conclusion}

A xylanase hyper-producing platform in T. reesei was established by constitutive expression of XYR1 and homologous expression of the native XYNII under the strong $c b h 1$ promoter. C30OExyr1/xyn2 $\Delta$ cbh 1 exhibited improved xylanase-producing ability using several carbon sources, $5256 \mathrm{U} / \mathrm{mL}$ xylanase activity and $9.25 \mathrm{U} /$ $\mathrm{mL} p$ NPXase activity were achieved on Avicel, associated with increased expression of several xylanolytic enzymes for efficient hydrolysis of xylan. Unexpectedly, deletion of ace1 in C30OExyr1 did not give any improvement, which might be the result of the disturbed function of the complex formed between ACE1 and XYR1. Besides, a considerable amount of xylanase production was achieved using economical and soluble carbon sources, showing great potential for industrial fermentation. In addition, a 34.63\% increase of APCS saccharification efficiency was attained using xylanase produced by C30OExyr1/ xyn $2 \Delta$ cbh 1 as accessory enzymes with $X / F=500$. The novel platform with outstanding ability for crude xylanase cocktail production would greatly fit in biomass degradation and give a new perspective of further engineering in T. reesei for industrial purposes.

\section{Methods}

\section{Strains and culture conditions}

Escherichia coli TOP10, used as a cloning host for plasmid construction, was cultured at $37^{\circ} \mathrm{C}$ in Luria-Bertani (LB) medium. Agrobacterium tumefaciens AGL1 was used as a media for the transformation of $T$. reesei and was cultured in LB medium or inducing medium (IM) at $28{ }^{\circ} \mathrm{C}$ according to purposes. T. reesei RUT-C30 (CICC 
13052) was a parent strain that was kindly provided by $\mathrm{Li}$ [34]. T. reesei C30 $\Delta$ ura3 was a uracil-deficient phenotype of RUT-C30 which was constructed previously in our lab. All the T. reese $i$ were cultured on potato dextrose agar (PDA) plate at $28{ }^{\circ} \mathrm{C}$ for conidiation. $10 \mathrm{mM}$ uracil was added when uracil-deficient $T$. reesei $\Delta$ ura3 was cultured. For enzyme induction, $0.5 \mathrm{~mL}$ conidia suspension $\left(10^{7} / \mathrm{mL}\right)$ was inoculated into $20 \mathrm{~mL}$ Sabouraud Dextrose Broth (SDB) and incubated for $40 \mathrm{~h}$ at $28^{\circ} \mathrm{C}$ with $200 \mathrm{rpm}$. After the accumulation of mycelia, the culture mixture was transferred into a $250 \mathrm{~mL}$ flask containing $50 \mathrm{~mL}$ of inducing medium with the inoculum rate of $10 \%(\mathrm{v} / \mathrm{v})$. The flask was routinely cultured at $28{ }^{\circ} \mathrm{C}$ with a constant shake of $200 \mathrm{rpm}$ for 5 days. The Avicelinducing medium was prepared as described previously [22], and wheat bran was added for sufficient nutrients. The glucose or lactose medium was similar to the Avicel-inducing medium that Avicel and wheat bran were replaced by lactose and glucose, respectively.

\section{Plasmid construction and fungal transformation}

All the recombinant plasmid was constructed based on pCAMBIA1301G, which was derived from pCAMBIA1301 by replacing the promoter of hygromycin-resistant gene from CaMV $35 S$ to a glyceraldehyde-3-phosphate dehydrogenase ( $g p d$ ) promoter of Aspergillus nidulans.

For overexpression of $x y r 1$ (jgi|Trire2:122208) and deletion of ace1 (jgi|Trire2:75418) in T. reesei, the xyr1 coding sequence was amplified from genomic DNA of RUT-C30 with primers listed in Additional file 1: Table S2, and inserted into the hyg locus of pCAMBIA1301G resulting in a $3.8-\mathrm{kb} x y r 1$ overexpression cassette. Then, the $1.3-\mathrm{kb}$ upstream and downstream of ace 1 was amplified from the genomic DNA of RUT-C30, combined with the xyr1 overexpression cassette and ura3 cassette, cloned to the backbone of pCAMBIA1301G using MultiF SeamLess Assembly Mix (ABclonal, Wuhan, China), resulting in pOExyr1 $\Delta$ ace1. All the vectors constructed above were verified by PCR and sequencing.

For overexpressing major endo-xylanase gene $x y n 2$ (jgi|Trire2:123818) of T. reesei under $c b h 1$ promoter, $0.7-\mathrm{kb} x y n 2$ coding sequence including signal peptide sequence was amplified from cDNA of RUT-C30 with primers xyn2F/R (Additional file 1: Table S2). The 1.3$\mathrm{kb}$ promoter and $1.1-\mathrm{kb}$ terminal sequence of $c b h 1$ were amplified from genomic DNA, and the resulting three sequences were ligated through overlap extension PCR using the primers Pcbh1F/Tcbh1R. The xyn2 expression cassette was ligated to the KpnI and SnaBI sites of linearized pCAMBIA1301G by T4 ligase (NEB, England), resulting in pCbhxyn2 (Fig. 4a). pPdcxyn2 was constructed by changing the $c b h 1$ promoter with $p d c$ promoter. To insert the expression cassette into $c b h 1$ locus, about $1.3-\mathrm{kb}$ upstream of the $c b h 1$ promoter was cloned into pCbhxyn2, resulting in pCbhxyn $2 \Delta \mathrm{cbh} 1$ (Fig. 4a).

The transformation of $T$. reesei was conducted by Agrobacterium tumefaciens-mediated transformation (ATMT) as described previously [52]. The selection of transformants was using MM plate [53] for uracil-deficient C30 4 ura3, and PDA containing $20 \mathrm{mg} / \mathrm{L}$ hygromycin for others. The putative transformants and correct insert of the cassette were verified by PCR, single spore was isolated through successively streaking. The recombinant strains mentioned in this paper were listed in Additional file 1: Table S1.

\section{Biochemical analysis}

Specifically, $1 \mathrm{~mL}$ mixture of shake flask fermentation was sampled every $24 \mathrm{~h}$, the sample was centrifuged at $4{ }^{\circ} \mathrm{C}, 12,000 \mathrm{rpm}$ for $10 \mathrm{~min}$ to remove hypha pellet, and the supernatant was transferred to another tube and filtered for further analysis. The protein concentration was determined by Bradford Protein Assay Kit (Beyotime Biotechnology, Shanghai, China) as indicated by manual instruction. Cellulase and xylanase activity was determined by DNS method $[16,54]$ with a few modifications. In brief, $6.6 \mathrm{mg}$ filter paper (Whatman No.1) was added into $180 \mu \mathrm{L}$ $50 \mathrm{mM}$ sodium citrate buffer with $\mathrm{pH}$ of 4.8 , then $20 \mu \mathrm{L}$ enzyme solution diluted to appropriate concentration was added to the mixture and incubated at $50{ }^{\circ} \mathrm{C}$ for $60 \mathrm{~min}$. The reaction was stopped by the addition of $300 \mu \mathrm{L}$ alkaline 3,5-dinitrosalicylic (DNS) and boiled for $5 \mathrm{~min}$, followed by immediate ice incubation. Then, the mixture was diluted fourfold and the absorbance at $540 \mathrm{~nm}$ was detected for correction of FPase activities. For xylanase activity determination, $180 \mu \mathrm{L} 1 \%$ oat spelt xylan (TCL, Japan) in $50 \mathrm{mM}$ sodium citrate buffer with $\mathrm{pH}$ of 5.3 was mixed with $20 \mu \mathrm{L}$ diluted enzyme and incubated for $5 \mathrm{~min}$, the following step was similar with cellulase activity analysis. One unit of enzyme activity was defined as the amount of enzyme that releases $1 \mu \mathrm{mol}$ reducing sugar per minute. For $\beta$-xylosidase and $\beta$-glucosidase activity assay, $90 \mu \mathrm{L}$ $4 \mathrm{mM} p \mathrm{NPX}$ or $p$ NPG in $50 \mathrm{mM}$ sodium acetate (pH 5.0) was mixed with $20 \mu \mathrm{L}$ appropriately diluted enzyme and incubated for $10 \mathrm{~min}$ at $50^{\circ} \mathrm{C}$. The reaction was stopped by adding $110 \mu \mathrm{L} 2 \%$ sodium carbonate, and then, absorbance at $410 \mathrm{~nm}$ was detected. One unit of enzyme activity was defined as the amount of enzyme that releases $1 \mu \mathrm{mol}$ $p$-nitrophenol per minute. SDS-PAGE analysis was performed on $9 \%$ Tris $-\mathrm{HCl}$ polyacrylamide gels using $3.75 \mu \mathrm{L}$ supernatant of different $T$. reesei strains. All experiments were performed in three biological replicates. 


\section{RNA extraction and RT-qPCR analysis}

Culture mixtures were sampled at different time, mycelia were collected by centrifuged at $4{ }^{\circ} \mathrm{C}, 12,000 \mathrm{rpm}$ for 10 min and washed twice with distilled water, then the pellet was rapidly frozen in liquid nitrogen and stored at $-80^{\circ} \mathrm{C}$ for further analysis. RNA extraction was conducted using RNAiso plus (Takara, Japan) according to the manual's instruction. The quality and quantity of extracted RNA were assessed by the absorbance at $260 \mathrm{~nm}$ on NanoDrop 8000 (Thermo Scientific, U.S.A.). Reverse transcription was performed using PrimeScript ${ }^{\mathrm{TM}}$ RT reagent Kit with gDNA Eraser (Perfect Real Time) (Takara, Japan) with total RNA extracted from different transformants cultured in inducing medium. Quantitative real-time PCR (RT-qPCR) was conducted in $20 \mu \mathrm{L}$ per tube with 2*SG Fast qPCR Master Mix (High Rox) (Sangon Biotech, Shanghai, China) using primers listed in Additional file 1: Table S3, and the transcription level of sar1 was used as inner standard. The PCR protocol run in the ABI StepOne instrument Plus (ABI, Germany) with software Version 2.3 (ABI, Germany) consisted of 3 min of initial denaturation at $95^{\circ} \mathrm{C}$, followed by 40 cycles of $3 \mathrm{~s}$ at $95{ }^{\circ} \mathrm{C}$ and $30 \mathrm{~s}$ at $60^{\circ} \mathrm{C}$. A melting curve was performed after each run to check the PCR product specificity. The data were calculated using $\left(2^{-\Delta \Delta C T}\right)$ for relative quantification. All PCRs were carried out in triplicate on a plate. upstream of ATG (the TATA box for $x y n 3$ was identified as TATA). For binding site exploration, the GGC(A/T) ${ }_{3}$ was searched upstream of the TATA box, the motif in two strands were both under consideration. For putative ACE1 binding site searching, the core element of AGGCA was used for identifying corresponded ACE1binding sites.

\section{Saccharification of alkali pretreated corn stover}

The corn stover was purchased from the country of Jiangsu (China), then, treated with $2 \% \mathrm{NaOH}$ with a load of $10 \%(\mathrm{w} / \mathrm{v})$ at $121{ }^{\circ} \mathrm{C}$ for $30 \mathrm{~min}$. The mixture was washed with distilled water until neutral $\mathrm{pH}$, and dried at $60^{\circ} \mathrm{C}$ for $24 \mathrm{~h}$, then smashed for better utilization. The alkali pretreated corn stover (APCS) was stored at $4{ }^{\circ} \mathrm{C}$ for further study. The content of APCS was determined using the NREL method [56], which contains $57.2 \%$ cellulose and $23.4 \%$ hemicellulose. The total activity of cellulase and xylanase was calculated through the formula below, and the total xylanase activity vs total cellulase activity was set as $X / F$ to evaluate the amount of cellulase and xylanase in each reaction. The lowest $X / F$ of 24 and highest of 2770 represented the sole reaction by cellulase and xylanase preparation, respectively. In addition, a series ratio from 24 to 2770 was set to systematically determine the best ratio for saccharification.

$$
\mathrm{X} / \mathrm{F}=\frac{\mathrm{Xylanase} \text { activity }_{\text {cellulase preparation }}+\text { Xylanase activity }_{\text {xylanase preparation }}}{\mathrm{FPU}_{\text {cellulase preparation }}+\mathrm{FPU}_{\text {xylanase preparation }}}
$$

\section{Copy number determination}

For copy number determination, the genomic DNA of strain was extracted and applied for qPCR with the previous method [55], the gene sar1 was represented as a single copy. The primer for copy number assay was also listed in Additional file: Table S3, with the qPCR procedure described above. Three biological replicates were performed for each reaction.

\section{In silico analysis of upstream of xylanolytic genes}

To give a comprehensive view of the regulation of the xylanolytic gene, the $5^{\prime}$ upstream region was searched for each gene using the $T$. reesei genome database v2.0 (https://genome.jgi.doe.gov/Trire2/Trire2.home. html). The $1000 \mathrm{bp}$ upstream from ATG of each gene was used for further analysis. The TATA box was identified by searching the TATAA motif about $100 \mathrm{bp}$
The commercial cellulase used in this study was kindly provided by Sunson (NingXia, China), and the xylanase cocktail was prepared by culturing C30OExyr $1 / x y n 2 \Delta$ cbh 1 in Avicel medium for 5 days. For saccharification experiments, $500 \pm 0.5 \mathrm{mg}$ APCS was mixed with $4 \mathrm{mg}$ (protein concentration) enzyme preparation (Table 2) in a $25 \mathrm{~mL}$ flask and finally making up to $10 \mathrm{~mL}$ with $50 \mathrm{mM}$ sodium acetate (pH 5.0). Besides, the $\beta$-glucosidase (Sunson) was added at CBU/ $F P U=2$ to limit the end-production inhibition. The reaction was performed at $50{ }^{\circ} \mathrm{C}, 150 \mathrm{rpm}$ for $72 \mathrm{~h}$. Samples were collected at 2, 6, 12, 24 and $72 \mathrm{~h}$, and the sugar content was analyzed by High-Performance Liquid Chromatography (HPLC) with a Bio-Rad Aminex HPX-87H column and Agilent 1260 Infinity II refractive index detector. $5 \mathrm{mM} \mathrm{H}_{2} \mathrm{SO}_{4}$ was used as mobile phase at a flow rate of $0.5 \mathrm{~mL} / \mathrm{min}$. All the experiments were conducted in three biological replicates. 


\section{Abbreviations}

APCS: Alkali pretreated corn stover; ATMT: Agrobacterium tumefaciensMediated transformation; CCR: Carbon catabolite repression; HPLC: HighPerformance Liquid Chromatography; MM: Minimal medium; RESS: REpression under Secretion Stress; SDB: Sabouraud Dextrose Broth; UPR: Unfolded Protein Response; XBD: XYR1-binding sites.

\section{Supplementary Information}

The online version contains supplementary material available at https://doi. org/10.1186/s13068-021-01943-9.

Additional file 1: Table S1. Strains used in this study. Table S2. Primers used for recombinant plasmid construction. Table S3. Primers used for RTqPCR and copy number determination. Table S4. Comparison of xylanase production in different organisms. Table S5. The hydrolysis efficiency of lignocellulose. Figure S1. The transcription level of xylanolytic genes of C30OExyr1/xyn2 $\Delta$ cbh1 in lactose and glucose medium. Figure S2. The transcription level of relative genes in C300Exyr1/xyn2 and C30OExyr1/ xyn $2 \Delta c b h 1$. Figure S3. The $\beta$-xylosidase ( $p$ NPXase) activity of the C30 $\triangle \mathrm{cbh} 1$ and the parent strain.

\section{Acknowledgements}

\section{Not applicable.}

\section{Authors' contributions}

SY, YX and XWY designed the study. SY conducted the experiments. SY and $X W Y$ analyzed the data and wrote the paper. All the authors read and approved the final manuscript.

\section{Funding}

This work was supported by Financial support from the National Natural Science Foundation of China (NSFC) (32072162); High-end Foreign Experts Recruitment Program (GDT20153200044); National First-Class Discipline Program of Light Industry Technology and Engineering (LITE2018-09); 111 Project (111-2-06) and Postgraduate Research \& Practice Innovation Program of Jiangsu Province (KYCX20_1819).

\section{Availability of data and materials}

All data generated or analyzed during this study are included in this published article and its Additional file 1 .

\section{Declarations}

Ethics approval and consent to participate Not applicable.

\section{Consent for publication}

Not applicable.

\section{Competing interests}

The authors declare that they have no known competing financial interests or personal relationships that could have appeared to influence the work reported in this paper.

Received: 19 January 2021 Accepted: 27 March 2021

Published online: 08 April 2021

\section{References}

1. Malgas S, Mafa MS, Mkabayi L, Pletschke BI. A mini review of xylanolytic enzymes with regards to their synergistic interactions during heteroxylan degradation. World J Microbiol Biotechnol. 2019;35:187.

2. Li C, Lin F, Li Y, Wei W, Wang H, Qin L, Zhou Z, Li B, Wu F, Chen Z. A betaglucosidase hyper-production Trichoderma reesei mutant reveals a potential role of cel3D in cellulase production. Microb Cell Fact. 2016;15:151.
3. Gao J, Qian YC, Wang YF, Qu YB, Zhong YH. Production of the versatile cellulase for cellulose bioconversion and cellulase inducer synthesis by genetic improvement of Trichoderma reesei. Biotechnol Biofuels. 2017;10:16.

4. Pauly M, Albersheim P, Darvill A, York WS. Molecular domains of the cellulose/xyloglucan network in the cell walls of higher plants. Plant J. 1999;20:629-39.

5. Chandra RP, Bura R, Mabee WE, Berlin A, Pan X, Saddler JN. Substrate pretreatment: the key to effective enzymatic hydrolysis of lignocellulosics? Biofuels. 2007;108:67-93.

6. Hu J, Arantes V, Saddler JN. The enhancement of enzymatic hydrolysis of lignocellulosic substrates by the addition of accessory enzymes such as xylanase: is it an additive or synergistic effect? Biotechnol Biofuels. 2011;4:36.

7. Bhardwaj N, Kumar B, Verma P. A detailed overview of xylanases: an emerging biomolecule for current and future prospective. Bioresour Bioprocess. 2019;6:36.

8. Walia A, Guleria S, Mehta P, Chauhan A, Parkash J. Microbial xylanases and their industrial application in pulp and paper biobleaching: a review. 3 Biotech. 2017;7:11.

9. Long LF, Zhang YB, Ren HY, Sun HY, Sun FBF, Qin WS. Recombinant expression of Aspergillus niger GH10 endo-xylanase in Pichia pastoris by constructing a double-plasmid co-expression system. J Chem Technol Biotechnol. 2020;95:535-43.

10. Paes G, Berrin JG, Beaugrand J. GH11 xylanases: Structure/function/properties relationships and applications. Biotechnol Adv. 2012;30:564-92.

11. Fonseca LM, Parreiras LS, Murakami MT. Rational engineering of the Trichoderma reesei RUT-C30 strain into an industrially relevant platform for cellulase production. Biotechnol Biofuels. 2020;13:93.

12. Wang J, Zeng D, Mai G, Liu G, Yu S. Homologous constitutive expression of Xyn III in Trichoderma reesei QM9414 and its characterization. Folia Microbiol. 2014;59:229-33.

13. Tenkanen M, Vrsanska M, Siika-aho M, Wong DW, Puchart V, Penttila M, Saloheimo M, Biely P. Xylanase XYN IV from Trichoderma reesei showing exo- and endo-xylanase activity. FEBS J. 2013;280:285-301.

14. Herold S, Bischof R, Metz B, Seiboth B, Kubicek CP. Xylanase gene transcription in Trichoderma reesei is triggered by different inducers representing different hemicellulosic pentose polymers. Eukaryot Cell. 2013;12:390-8.

15. Kristufek D, Zeilinger $S$, Kubicek $C$. Regulation of $\beta$-xylosidase formation by xylose in Trichoderma reesei. Appl Microbiol Biotechnol. 1995;42:713-7.

16. Torronen A, Mach RL, Messner R, Gonzalez R, Kalkkinen N, Harkki A, Kubicek CP. The two major xylanases from Trichoderma reesei: characterization of both enzymes and genes. Nat Biotechnol. 1992;10:1461-5.

17. Zouari Ayadi D, Hmida Sayari A, Ben Hlima H, Ben Mabrouk S, Mezghani M, Bejar S. Improvement of Trichoderma reesei xylanase II thermal stability by serine to threonine surface mutations. Int J Biol Macromol. 2015;72:163-70.

18. Portnoy $T$, Margeot $A$, Linke R, Atanasova L, Fekete $E$, Sandor E, Hartl L, Karaffa L, Druzhinina IS, Seiboth B, et al. The CRE1 carbon catabolite repressor of the fungus Trichoderma reesei: a master regulator of carbon assimilation. BMC Genom. 2011;12:269.

19. Stricker $A R$, Steiger MG, Mach RL. Xyr1 receives the lactose induction signal and regulates lactose metabolism in Hypocrea jecorina. FEBS Lett. 2007:581:3915-20.

20. Aro N, Ilmen M, Saloheimo A, Penttila M. ACEl of Trichoderma reesei is a repressor of cellulase and xylanase expression. Appl Environ Microbiol. 2003;69:56-65.

21. Derntl C, Rassinger A, Srebotnik E, Mach RL, Mach-Aigner AR. Xpp1 regulates the expression of xylanases, but not of cellulases in Trichoderma reesei. Biotechnol Biofuels. 2015;8:112.

22. Liu R, Chen L, Jiang Y, Zou G, Zhou Z. A novel transcription factor specifically regulates $\mathrm{GH} 11$ xylanase genes in Trichoderma reesei. Biotechnol Biofuels. 2017;10:194.

23. Saarelainen R, Paloheimo M, Fagerstrom R, Suominen PL, Nevalainen KM. Cloning, sequencing and enhanced expression of the Trichoderma reesei endoxylanase II (pl 9) gene xIn2. Mol Gen Genet. 1993;241:497-503.

24. de Faria FP, Te OV, Bergquist PL, Azevedo MO, Nevalainen KM. Expression and processing of a major xylanase (XYN2) from the thermophilic fungus Humicola grisea var. thermoidea in Trichoderma reesei. Lett Appl Microbiol. 2002;34:119-23. 
25. Miyauchi S, Te'o VS Jr, Bergquist PL, Nevalainen KM. Expression of a bacterial xylanase in Trichoderma reesei under the egl2 and cbh2 glycosyl hydrolase gene promoters. N Biotechnol. 2013;30:523-30.

26. Sun $X$, Zhang $X$, Huang $H$, Wang $Y, T u T$, Bai $Y$, Wang $Y$, Z Zhang J, Luo H, Yao $B$, et al. Engineering the cbh1 Promoter of Trichoderma reesei for enhanced protein production by replacing the binding sites of a transcription repressor ACE1 to those of the activators. J Agric Food Chem. 2020;68:1337-46.

27. Xue X, Wu Y, Qin X, Ma R, Luo H, Su X, Yao B. Revisiting overexpression of a heterologous beta-glucosidase in Trichoderma reesei: fusion expression of the Neosartorya fischeri Bgl3A to cbh1 enhances the overall as well as individual cellulase activities. Microb Cell Fact. 2016;15:122.

28. Li J, Wang J, Wang S, Xing M, Yu S, Liu G. Achieving efficient protein expression in Trichoderma reesei by using strong constitutive promoters. Microb Cell Fact. 2012;11:84.

29. Rauscher R, Wurleitner E, Wacenovsky C, Aro N, Stricker AR, Zeilinger S, Kubicek CP, Penttila M, Mach RL. Transcriptional regulation of xyn1, encoding xylanase I, in Hypocrea jecorina. Eukaryot Cell. 2006:5:447-56.

30. Mach-Aigner AR, Pucher ME, Steiger MG, Bauer GE, Preis SJ, Mach RL. Transcriptional regulation of xyr1, encoding the main regulator of the xylanolytic and cellulolytic enzyme system in Hypocrea jecorina. Appl Environ Microbiol. 2008;74:6554-62.

31. Derntl C, Gudynaite-Savitch L, Calixte S, White T, Mach RL, Mach-Aigner AR. Mutation of the Xylanase regulator 1 causes a glucose blind hydrolase expressing phenotype in industrially used Trichoderma strains. Biotechnol Biofuels. 2013;6:62.

32. Furukawa T, Shida Y, Kitagami N, Mori K, Kato M, Kobayashi T, Okada H, Ogasawara W, Morikawa Y. Identification of specific binding sites for XYR1, a transcriptional activator of cellulolytic and xylanolytic genes in Trichoderma reesei. Fungal Genet Biol. 2009;46:564-74.

33. Qin LN, Jiang XZ, Dong ZY, Huang JZ, Chen XZ. Identification of two integration sites in favor of transgene expression in Trichoderma reesei. Biotechnol Biofuels. 2018;11:1-5.

34. Li C, Lin F, Zhou L, Qin L, Li B, Zhou Z, Jin M, Chen Z. Cellulase hyperproduction by Trichoderma reesei mutant SEU-7 on lactose. Biotechnol Biofuels. 2017;10:228.

35. Choudhary J, Saritha M, Nain L, Arora A. Enhanced saccharification of steam-pretreated rice straw by commercial cellulases supplemented with xylanase. J Bioprocess Biotech. 2014;4:1.

36. Wang K, Cao R, Wang M, Lin Q, Zhan R, Xu H, Wang S. A novel thermostable GH10 xylanase with activities on a wide variety of cellulosic substrates from a xylanolytic Bacillus strain exhibiting significant synergy with commercial Celluclast $1.5 \mathrm{~L}$ in pretreated corn stover hydrolysis. Biotechnol Biofuels. 2019;12:48

37. Bajaj P, Mahajan R. Cellulase and xylanase synergism in industrial biotechnology. Appl Microbiol Biotechnol. 2019;103:8711-24.

38. Wang S, Liu G, Wang J, Yu J, Huang B, Xing M. Enhancing cellulase production in Trichoderma reesei RUT C30 through combined manipulation of activating and repressing genes. J Ind Microbiol Biotechnol. 2013;40:633-41.

39. Chen L, Zou G, Wang J, Wang J, Liu R, Jiang Y, Zhao G, Zhou Z. Characterization of the $\mathrm{Ca}(2+)$-responsive signaling pathway in regulating the expression and secretion of cellulases in Trichoderma reesei Rut-C30. Mol Microbiol. 2016;100:560-75.

40. Hahn S, Young ET. Transcriptional regulation in Saccharomyces cerevisiae: transcription factor regulation and function, mechanisms of initiation, and roles of activators and coactivators. Genetics. 2011;189:705-36.

41. Furukawa T, Shida Y, Kitagami N, Ota Y, Adachi M, Nakagawa S, Shimada R, Kato M, Kobayashi T, Okada H, et al. Identification of the cis-acting elements involved in regulation of xylanase III gene expression in Trichoderma reesei PC-3-7. Fungal Genet Biol. 2008;45:1094-102.

42. Zhang J, Chen Y, Wu C, Liu P, Wang W, Wei D. The transcription factor ACE3 controls cellulase activities and lactose metabolism via two additional regulators in the fungus Trichoderma reesei. J Biol Chem. 2019;294:18435-50.
43. Wurleitner E, Pera L, Wacenovsky C, Cziferszky A, Zeilinger S, Kubicek CP, Mach RL. Transcriptional regulation of xyn2 in Hypocrea jecorina. Eukaryot Cell. 2003;2:150-8.

44. Singh A, Taylor LE 2nd, Vander Wall TA, Linger J, Himmel ME, Podkaminer K, Adney WS, Decker SR. Heterologous protein expression in Hypocrea jecorina: a historical perspective and new developments. Biotechnol Adv. 2015;33:142-54.

45. Qin LN, Cai FR, Dong XR, Huang ZB, Tao Y, Huang JZ, Dong ZY. Improved production of heterologous lipase in Trichoderma reesei by RNAi mediated gene silencing of an endogenic highly expressed gene. Bioresour Technol. 2012;109:116-22.

46. Al-Sheikh H, Watson AJ, Lacey GA, Punt PJ, MacKenzie DA, Jeenes DJ, Pakula T, Penttilä M, Alcocer MJ, Archer DB. Endoplasmic reticulum stress leads to the selective transcriptional downregulation of the glucoamylase gene in Aspergillus niger. Mol Microbiol. 2004;53:1731-42.

47. Fan F, Ma G, Li J, Liu Q, Benz JP, Tian C, Ma Y. Genome-wide analysis of the endoplasmic reticulum stress response during lignocellulase production in Neurospora crassa. Biotechnol Biofuels. 2015;8:66.

48. Ye Y, Li X, Cao Y, Du J, Chen S, Zhao J. A beta-xylosidase hyper-production Penicillium oxalicum mutant enhanced ethanol production from alkalipretreated corn stover. Bioresour Technol. 2017;245:734-42.

49. Shibata $N$, Suetsugu M, Kakeshita $H$, Igarashi $K$, Hagihara $H$, Takimura Y. A novel GH10 xylanase from Penicillium sp accelerates saccharification of alkaline-pretreated bagasse by an enzyme from recombinant Trichoderma reesei expressing Aspergillus beta-glucosidase. Biotechnol Biofuels. 2017;10:17.

50. Husson E, Auxenfans T, Herbaut M, Baralle M, Lambertyn V, Rakotoarivonina H, Remond C, Sarazin C. Sequential and simultaneous strategies for biorefining of wheat straw using room temperature ionic liquids, xylanases and cellulases. Bioresour Technol. 2018;251:280-7.

51. Basit A, Liu J, Miao T, Zheng F, Rahim K, Lou H, Jiang W. Characterization of Two Endo-beta-1, 4-Xylanases from Myceliophthora thermophila and Their Saccharification Efficiencies, Synergistic with Commercial Cellulase. Front Microbiol. 2018;9:233.

52. Zhu SY, Xu Y, Yu XW. Improved homologous expression of the acidic lipase from Aspergillus niger. J Microbiol Biotechnol. 2020;30:196-205.

53. Penttilä M, Nevalainen H, Rättö M, Salminen E, Knowles J. A versatile transformation system for the cellulolytic filamentous fungus Trichoderma reesei. Gene. 1987;61:155-64.

54. Ghose TK. Measurement of cellulase activities. Pure Appl Chem. 1987:59:257-68.

55. Solomon PS, Ipcho SV, Hane JK, Tan K-C, Oliver RP. A quantitative PCR approach to determine gene copy number. Fungal Genet Rep. 2008;55:5-8.

56. Sluiter AD, Hames BR, Ruiz RE, Scarlata CJ, Sluiter JB, Templeton DW, Crocker DP. Determination of structural carbohydrates and lignin in biomass. Lab Anal Proced. 2008;1617:1-16.

\section{Publisher's Note}

Springer Nature remains neutral with regard to jurisdictional claims in published maps and institutional affiliations. 